\title{
Genome information management and integrated data analysis with HaloLex
}

\author{
Friedhelm Pfeiffer • Alexander Broicher • \\ Thomas Gillich · Kathrin Klee · José Mejía . \\ Markus Rampp $\cdot$ Dieter Oesterhelt
}

Received: 7 January 2008/Revised: 1 April 2008/Accepted: 8 May 2008/Published online: 1 July 2008

(C) The Author(s) 2008

\begin{abstract}
HaloLex is a software system for the central management, integration, curation, and web-based visualization of genomic and other -omics data for any given microorganism. The system has been employed for the manual curation of three haloarchaeal genomes, namely Halobacterium salinarum (strain R1), Natronomonas pharaonis, and Haloquadratum walsbyi. HaloLex, in particular, enables the integrated analysis of genome-wide proteomic results with the underlying genomic data. This has proven indispensable to generate reliable gene predictions for GC-rich genomes, which, due to their characteristically low abundance of stop codons, are known to be hard targets for standard gene finders, especially concerning start codon assignment. The proteomic identification of more than $600 \mathrm{~N}$-terminal peptides has greatly increased the reliability of the start codon assignment for Halobacterium salinarum. Application of homology-based methods to the published genome of Haloarcula marismortui allowed to detect 47 previously unidentified genes (a problem that is particularly serious for short protein sequences) and to correct more than 300 start codon misassignments.
\end{abstract}

Communicated by Erko Stackebrandt.

F. Pfeiffer - A. Broicher · T. Gillich · K. Klee .

D. Oesterhelt $(\square)$

Department of Membrane Biochemistry,

Max-Planck-Institute of Biochemistry,

Am Klopferspitz 18, 82152 Martinsried, Germany

e-mail: oesterhe@biochem.mpg.de

J. Mejía $\cdot$ M. Rampp

Computing Center (RZG) of the Max-Planck-Society,

Max Planck Institute of Plasma Physics,

Boltzmannstrasse 2, 85748 Garching, Germany
Keywords Halophilic archaea .

Genome information system - Genome browser .

Proteomics - Biological data curation .

Start codon assignment · Dinucleotide bias

\section{Introduction}

In the era of high-throughput biochemical experiments and large-scale systems-modeling approaches, the availability of high-quality input data like the complete gene or protein inventory of an organism is of paramount importance (cf. Kitano 2002). In practice, however, progress is often hampered by the lack of access to the relevant data, their insufficient integration with related information, or simply inadequate reliability of the data. Although the current era is commonly referred to as "postgenomic," many problems related to the quality of (microbial) genome annotations are still not satisfactorily solved. The GC-rich genomes of halophilic archaea, for example, are known to pose particular challenges for the bioinformatic prediction of their gene and protein inventory. Unsupervised, automatic gene prediction likely fails and blindly relying on such data can apparently compromise any further analysis or experiment. Thus, there is not only a need for making genomic and other related data available to the end-user in a most convenient and comprehensive way, but also tools are required that support generating, managing, and manually curating the data and allow experts to assess and improve their quality.

To this end, we have developed HaloLex, which serves both the aforementioned purposes. Halolex is a software system for the central management, integration, and webbased visualization of genomic and other -omics data for any given microorganism. Centered on the genomic information, HaloLex provides a comprehensive and user- 
friendly web interface (http://www.halolex.mpg.de) with many different interlinked views and various search functionalities to the underlying database. Advanced data mining tasks can be performed by employing high-level programming interfaces to access and automatically process bulk-data with computer scripts and programs.

The main scientific purpose of HaloLex is to support indepth analysis of selected prokaryotic genomes and to assist knowledge-based manual revision and refinement of their annotation, in particular by taking into account also nongenomic, experimental data (e.g., proteomics). Typically, genomic data enter the system after automatic gene identification, classification, and basic annotations have been accomplished using a general-purpose genome annotation system like, e.g., "GenDB" (Meyer et al. 2003).

We are not trying to parallel seemingly similar efforts like the "integrated microbial genomes browser IMG" (Markowitz et al. 2006), the "UCSC Archaeal Genome Browser" (Schneider et al. 2006) or "PEDANT" (Riley et al. 2007), "AGMIAL" (Bryson et al. 2006), or alike (see Bryson et al. 2006 for a recent overview), which are fullblown (automatic) genome annotation and/or information systems and provide exhaustive data repositories to the community. The focus of the HaloLex system is rather to assist experts in achieving an extraordinarily high data quality for a selection of (model) organisms and to make that data available for further analysis like systems modeling, experiment design, etc. To this end, we place particular emphasis on integrating standard genomic data with proteomic (see also Pleissner et al. 2004), transcriptomic, and metabolomic data. This has, for example, enabled a number of genome-scale proteomics (Tebbe et al. 2005; Klein et al. 2005; Bisle et al. 2006; Falb et al. 2006; Aivaliotis et al. 2007; Konstantinidis et al. 2007) and transcriptomic (Twellmeyer et al. 2007) analyses as well as a whole genome metabolic flux simulation (Gonzalez et al. 2008).

So far, scientific applications with HaloLex have mainly been focussing on a number of halophilic archaea, in particular on Halobacterium salinarum strain RI (DSM 671, Pfeiffer et al. 2008), Natronomonas pharaonis strain $G a$ bara (DSM 2160, Falb et al. 2005), and Haloquadratum walsbyi strain HBSQ001 (DSM 16790, Bolhuis et al. 2006). These genomes, together with Halobacterium salinarum strain NRC-1 (Ng et al. 2000), Haloarcula marismortui (Baliga et al. 2004) and Haloferax volcanii (J. Eisen, unpublished), are of primary interest to our own group and our collaborators. For specific examples, the reader is referred to the articles of Teufel et al. (2008), Scheuch et al. (2008), Dambeck and Soppa (2008) (all three references in this issue of Archives of Microbiology), and to the general review on the genomics and functional genomics of halophilic archaea by Soppa et al. (2008) (this issue of Archives of Microbiology).
However, HaloLex is not limited to halophilic archaea but currently covers all publicly available archaeal and a few selected bacterial genomes as obtained from the NCBI (ftp.ncbi.nih.gov/genomes/Bacteria). Besides demonstrating that the system is in principle capable of handling data for a large number of organisms, it allows users to browse the underlying GenBank data (augmented with additional information like bioinformatic predictions) within the same, coherent web interface. The integrated access within the same data model and software environment has shown to be a key prerequisite for conducting comprehensive statistical analyses like the ones presented in the second part of this paper.

The paper is organized as follows: in "Section 1: overview of the HaloLex system", we describe the main functionalities of the HaloLex system and give some notes on its implementation. "Section 2: integrated data analysis with HaloLex" highlights a number of biological problems that have been addressed with HaloLex, and points out bioinformatic solutions for the specific challenges posed by the GC-rich genomes of halophilic archaea. In particular, we shall present new, significantly improved annotation data for Haloarcula marismortui.

\section{Section 1: overview of the HaloLex system}

In short, HaloLex is based on a relational database serving as the central repository for all kinds of data, which are available for a given microorganism. A dynamic web application provides integrated access to the data and supports the daily work with the genomic and proteomic information in an economical way. The web interface is complemented by a programming interface, which enables (computationally experienced) local users to perform complex data mining tasks, based on a coherent data model and query methods.

Main functionalities of the web application

The primary and most accessible interface to the data stored in HaloLex is a web application, which allows to conveniently browse and query data over the Internet with a minimum technical effort (http://www.halolex.mpg.de). Depending on their individual role, anonymous or appropriately authorized users get read-only access to various browsing and search functionalities or are equipped with additional privileges for data curation and management, respectively. Access rights can be granted separately for each individual strain allowing us to handle all data within the same data store and code base.

Wherever applicable, graphics are rendered in the SVG (Scalable Vector Graphics) format. This greatly facilitates 
postprocessing of results and improves the quality of their presentation as compared to working with pixel-based formats (GIF, JPEG, PNG, etc.), which are conventionally employed by the majority of existing web applications.

\section{Genome viewer}

The available information about an individual coding sequence is summarized by a central "details page" listing sequences (coding region and protein translation), functional information (e.g., protein name, gene name, EC number, functional classification), general gene and protein characteristics (e.g., sequence length, start and stop codons, GC content, theoretical pI value), and results from several bioinformatic tools, e.g., transmembrane and signal peptide prediction with "Phobius" (Kall et al. 2004), protein export signals with "Tatfind" (Rose et al. 2002), codon adaptation index (Sharp and Li 1987), etc. In addition, the details page shows homologous sequences as well as cross-references to entries of the same protein in major public sequence databases like GenBank, UniProt, Kegg, and also links to relevant PubMed abstracts.

Usually, the details page is reached by selecting an organism and directly specifying an identifier or name for the gene of interest. In addition, also less specific searches and browsing functionalities are supported, including the option to obtain complete lists of genes or proteins, which can optionally be filtered by various characteristics like $\mathrm{pI}$ value range, type of proteomic identification, etc. (cf. Fig. 1).

If the organism or gene of interest is not specified a priori, the user can alternatively start out with a blastbased search (Altschul et al. 1997) for all sequences in the HaloLex database, which are similar to a given query.

To reach the details page, one may also start from a graphical display of a particular region on the genome. The corresponding "region viewer" page provides standard genome browsing functionalities and allows to color-code genes according to a variety of characteristics like the annotation status, assigned function class, GC content, proteomic identification (see Fig. 2), and many more.

\section{Genome curation}

For the manual curation of genome-based data, the web interface provides basic forms for updating the protein function annotation of individual genes (i.e., changing protein name, gene name, EC number, etc.).

In addition, the gene assignment itself can be revised. HaloLex supports the introduction of newly identified genes, which, e.g., may have been missed by some automatic gene prediction tool. Such tools may also have produced false positives, i.e., open reading frames (ORFs) that are eventually found not to code for proteins. Such "spurious ORFs", which are especially frequent in GC-rich genomes (cf. "Section 2: integrated data analysis with HaloLex"), are not eliminated from the database but get appropriately tagged. This allows to optionally retain such ORFs in viewing and data mining tools (cf. Fig. 2).

Furthermore, start codons may have been misassigned, which is also a common problem for GC-rich genomes (cf. "Section 2: integrated data analysis with HaloLex"). HaloLex assists the curator in assessing and revising the setting of the start codon by showing a number of characteristic quantities like the resulting amino-acid distribution or $\mathrm{pI}$ values corresponding to all relevant alternative choices of the start codon.

\section{Viewers for proteomic data}

Figure 3 illustrates a navigation path from a spot on a twodimensional gel image via the spectrum taken in a massspectrometric experiment to the identified protein. Individual spots on the gel image, for which spectra have been taken, are classified and color-coded according to the type and quality of the protein identification. The corresponding mass-intensity spectra are annotated and rendered such that the interpretation of the "raw" spectrum immediately gets transparent for the user.

\section{Data mining capabilities}

Naturally, not all conceivable types of data analysis can be anticipated and implemented in a web application with limited effort. For example, we opted not to provide sophisticated web-based cross-genome comparison functionalities. To still support complex and highly customizable data mining applications, HaloLex offers full programmatic access to all data and tools within a wellstructured data model. Being able to work in such a coherent environment has proven to be a fundamental prerequisite for a large variety of research projects, which have been conducted with HaloLex in the course of several years, a few current examples of which shall be highlighted in the subsequent section. The corresponding application programming interface (API) requires analysis programs to be written in the Java language and to run in the same localarea network where the HaloLex server is located. Both restrictions can, however, be relaxed by means of a SOAPbased web service interface, which we are internally already employing successfully (for a nontechnical introduction to web services and their role in biosciences, see Stein 2002).

Integration of other -omics data

The HaloLex database allows storing and accessing other omics data in an integrated way and links them with the 
Search Proteins By Proteomics Results

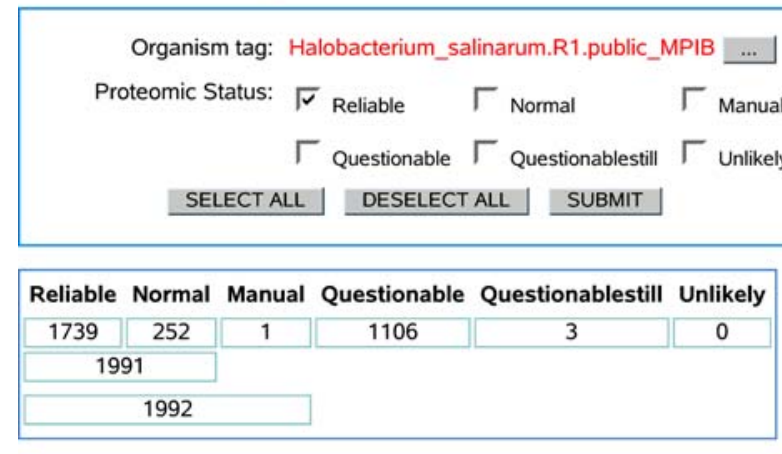

Number of hits found: 1739

\begin{tabular}{|c|c|c|c|c|c|c|c|}
\hline$\underline{\mathrm{ID} t}$ & Gene & $\underline{F C}$ & Protein name & Contig & Position & Rstatus & $\underline{\text { MS }}$ \\
\hline OE1001F & - & $\mathrm{CHY}$ & conserved hypothetical protein & $\mathrm{CHR}$ & $\underline{248-1453 F}$ & ok & Reliable \\
\hline OE1004F & - & & ABC-type transport system ATP-binding protein & $\mathrm{CHR}$ & $1450-2115 \mathrm{~F}$ & ok & Reliable \\
\hline OE1005F & - & TP & ABC-type transport system permease protein & $\mathrm{CHR}$ & $\underline{2112-3254 F}$ & ok & Reliable \\
\hline OE1008F & - & GEN & homolog to oligosaccharyl transferase & $\mathrm{CHR}$ & $3322-5643 \mathrm{~F}$ & ok & Reliable \\
\hline OE1013R & glms & $\mathrm{CHM}$ & $\begin{array}{l}\text { glutamine--fructose-6-phosphate aminotransferase (isomerizing) } \\
\text { (EC 2.6.1.16) }\end{array}$ & $\mathrm{CHR}$ & $\underline{5646-7451 R}$ & ok & Reliable \\
\hline OE1014R & graD5 & $\mathrm{CHM}$ & sugar nucleotidyltransferase (EC 2.7.7.-) & $\mathrm{CHR}$ & $\underline{7454-8641 R}$ & ok & Reliable \\
\hline OE1016R & graD2 & $\mathrm{CHM}$ & sugar nucleotidyltransferase (EC 2.7.7.-) & $\mathrm{CHR}$ & $\underline{8655-9860 R}$ & ok & Reliable \\
\hline OE1019R & - & ISH & IS1341-type transposase (TCE32) & $\mathrm{CHR}$ & $11478-12734 R$ & ok & Reliable \\
\hline OE1022R & - & $\mathrm{CHY}$ & conserved hypothetical protein & $\mathrm{CHR}$ & $13462-13812 R$ & ok & Reliable \\
\hline OE1023R & - & $\mathrm{CHY}$ & conserved hypothetical protein & $\mathrm{CHR}$ & 13809-14201R & ok & Reliable \\
\hline OE1062F & - & $\mathrm{CHY}$ & conserved hypothetical protein & $\mathrm{CHR}$ & $32104-32358 \mathrm{~F}$ & ok & Reliable \\
\hline OE1067R & - & $\mathrm{CHY}$ & conserved hypothetical protein & $\mathrm{CHR}$ & $33613-33918 \mathrm{R}$ & ok & Reliable \\
\hline OE1077R & ugd2 & $\mathrm{CHM}$ & UDP-glucose 6-dehydrogenase (EC 1.1.1.22) & $\mathrm{CHR}$ & 40626-41918R & ok & Reliable \\
\hline OE1078F & graD6 & $\mathrm{CHM}$ & sugar nucleotidyltransferase (EC 2.7.7.-) & $\mathrm{CHR}$ & 42006-42734F & ok & Reliable \\
\hline OE1079F & - & $\mathrm{CHY}$ & conserved hypothetical protein & $\mathrm{CHR}$ & 42895-43674F & ok & Reliable \\
\hline OE1081R & gth6 & GEN & probable glycosyltransferase, type 1 & $\mathrm{CHR}$ & $45260-46471 R$ & ok & Reliable \\
\hline
\end{tabular}

Fig. 1 Screenshot of the search functionality of HaloLex. Example output of a query for all genes of Halobacterium salinarum (R1), which were "reliably" identified by proteomics (indicated in the rightmost column). The complete list of 1,992 identifications was truncated for brevity

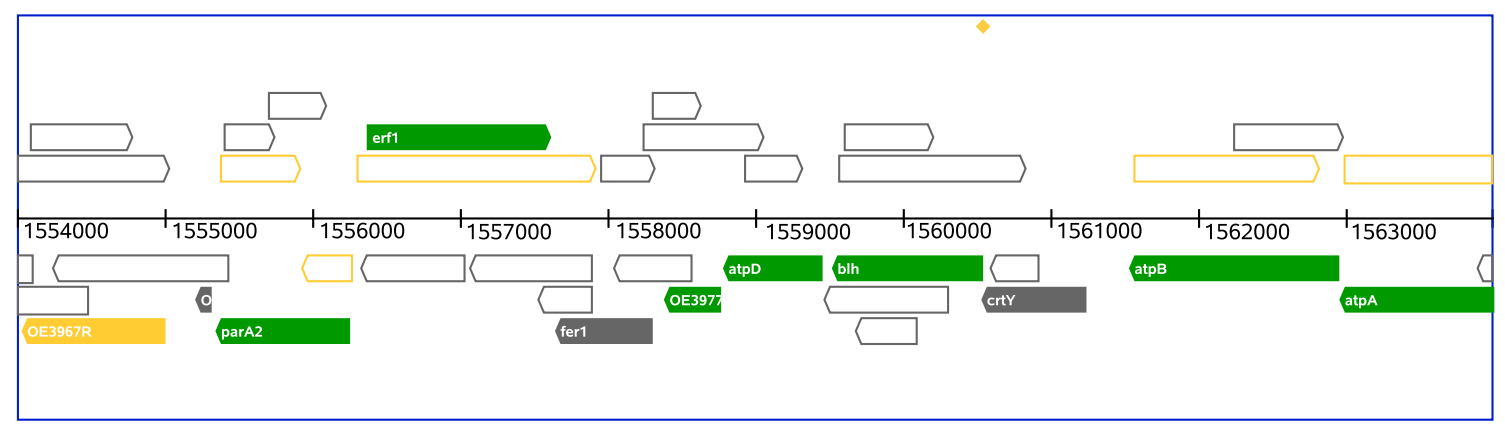

Fig. 2 Screenshot of the region viewer of HaloLex. Genomic region on the Halobacterium chromosome with ORFs color-coded according to different trust levels of proteomic identification. "Spurious" ORFs (which are hidden by default) are rendered as open symbols

corresponding genomic data. As shown above, this is well established for proteomic data (currently limited to database searches using MASCOT) and also applies to transcriptomic (Twellmeyer et al. 2007), as well as to curated metabolic data based on KEGG information (Falb et al. 2008). Access to the latter, however, is currently restricted to internal data mining applications, i.e., transcriptomic and metabolic data have not yet been made publicly available via the HaloLex web interface.
Notes on the implementation

HaloLex was originally implemented as a classic "LAMP" system, i.e., it has been operated on a Linux platform, using an Apache webserver, the Mysql relational database management system, and employing the Perl programming language. The system has been mainly used for department-internal purposes and covered only a few genomes. 


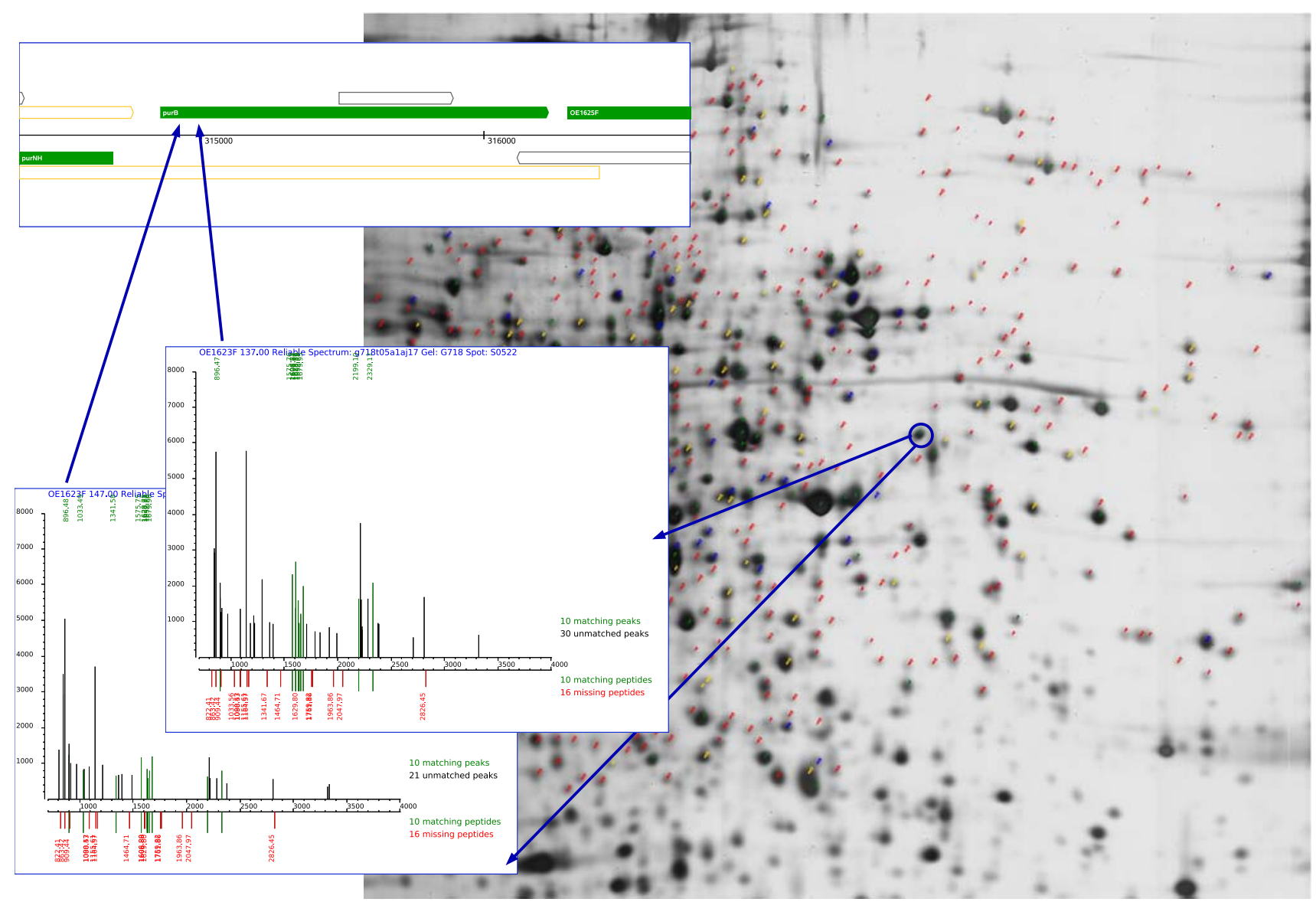

Fig. 3 Integrated access to genomic and proteomic data. Montage of different views of the HaloLex web interface on proteomic data. Blue arrows indicate example navigation tracks from a particular spot on a

To substantially extend the system with respect to the amount and complexity of data and to provide user-friendly public web access to the wealth of internal HaloLex functionalities, the system was recently reimplemented based on the Java Enterprise Edition 5 platform (see, e.g., Stearns et al. 2006). Besides many other well-established benefits delivered by this technology, we take advantage of the so-called "distributed components" approach, which promotes (loose) coupling of different stand-alone services through standardized interfaces. Specifically, HaloLex uses remote services offered by the MIGenAS sequence analysis platform (Rampp et al. 2006), e.g., for computing bioinformatic predictions like the transmembrane topology and for cross-referencing database identifiers (cf. Wu et al. 2004). For genome sequences imported from GenBank, we employ the SIMAP web service (Rattei et al. 2006) to retrieve precalculated and regularly updated similarities of proteins with public sequence databases like UniProt, PDB, etc. Data mining applications are enabled by an Application Programming Interface (API), which is built upon the "Remote Interface" component of Java's Enterprise
2D gel image via two different mass-spectra to the identified protein, and its location on the genome, respectively

Edition. The same technology is easily exploited to export a web service interface.

\section{Section 2: integrated data analysis with HaloLex}

A typical gene prediction problem, ORF overprediction, was chosen as a principal topic to illustrate several applications of HaloLex. We describe the statistical basis for this problem and how an integrated analysis of proteomic and genomic data allows to overcome it. In addition, we describe homology-based methods to detect and resolve gene prediction problems. Using the manual curation tools of HaloLex, we were able to substantially improve the gene prediction for the published genome of Haloarcula marismortui (Baliga et al. 2004).

GC-rich genomes like those of halophilic archaea are known to challenge standard gene prediction tools (Nielsen and Krogh 2005; McHardy et al. 2004). Two types of problems are encountered: (1) the existence of alternative long open reading frames (Veloso et al. 2005) makes it 
difficult to discriminate protein-coding genes from spurious ORFs; (2) start codon selection is highly error-prone due to long N-terminal ORF extensions in front of the start codon used in vivo (Aivaliotis et al. 2007). In both cases, which we summarize as the "ORF overprediction problem", noncoding DNA may be erroneously "translated" into protein sequences upon unwary application of gene predictors. This markedly deteriorates the quality of the resulting protein-coding gene set. A high-quality gene set is, however, essential for genetic experiments, analysis of transcription and translation signals, or the analysis of protein export signals, which are commonly located in the $\mathrm{N}$-terminal region, not to speak of systems biology applications such as metabolic modeling.

ORF overprediction is illustrated in Fig. 2, which shows a $10 \mathrm{~kb}$ region of the Halobacterium salinarum strain R1 genome. Protein-coding genes are outnumbered by spurious ORFs, which are all longer than 100 codons. In many cases, a spurious ORF is even longer than the proteincoding gene with which it overlaps. Spurious ORFs with a length of up to 1,300 codons have been found in the Halobacterium genome (Pfeiffer et al. 2008).

The ORF overprediction problem is also strikingly illustrated by the fact that $20 \%$ of the predicted protein sequences of strain NRC-1 of Halobacterium salinarum are inconsistent with those of strain R1, although the DNA sequences of both strains are virtually identical (four single-base differences, five one-base frameshifts, three indels; see Pfeiffer et al. 2008). Among the genes with a start codon assignment discrepancy is the TATA-binding protein tbpA (Scheuch et al. 2008).

\section{Genome statistical data}

ORF overprediction is caused by the low number of stop codons in GC-rich genomes (Veloso et al. 2005). Because of the reduced frequencies of $\mathrm{T}$ and $\mathrm{A}$, there is a low expectation value for each of the three stop codons (TAA, TAG, and TGA). The problem is further aggravated, because the number of stop codons actually found in prokaryotic genomes is even lower than that predicted by basic statistics of single-nucleotide frequencies. In case of Halobacterium, only $66 \%$ of the statistically expected stop codons are found. It is interesting to note that nearly all prokaryotic genomes have less stop codons than expected (Fig. 4). While the reduction is moderate for AT-rich genomes, it is significant for genomes with a GC content larger than $60 \%$, where $28 \%$ of the expected stop codons are missing on average.

This observation can be explained by an additional bias at the dinucleotide level, which exists on top of the aforementioned bias due to an altered GC content. For the Halobacterium chromosome, as an example, this is

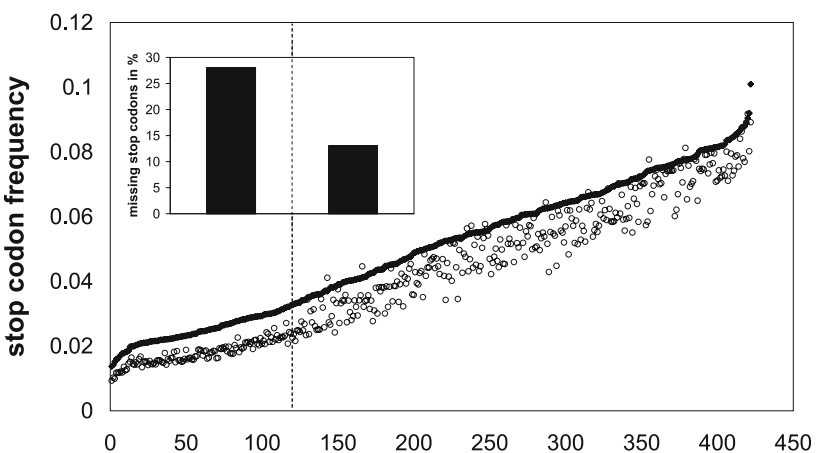

Fig. 4 Expected and actual frequency of stop codons for 425 microbial genomes. For the chromosomes of 425 microbial strains, the expected and the actual number of stop codons was counted and normalized by the total number of codons. Species are sorted along the abscissa by decreasing GC content. For nearly all genomes, the number of actually present stop codons (open circles) is significantly lower than that expected (filled symbols). The small inset shows that for the group of genomes with a GC content $>60 \%$ (to the left of the dashed vertical line), only $72 \%$ of the expected stop codons are found, whereas more than $85 \%$ of the expected stop codons are actually present in the group of genomes with a GC content $<60 \%$ (to the right of the dashed vertical line). The GenBank data for all microbial strains were downloaded from ftp.ncbi.nih.gov/genomes/ Bacteria. Only the chromosome (more precisely: the longest replicon) was chosen for each strain and only one representative strain was used for each species

illustrated by Fig. 5a, which shows that dinucleotides with the same number of $\mathrm{A}$ or $\mathrm{T}$ residues do not occur with equal frequencies. In particular, the "TA" dinucleotide, which appears in two of the three stop codons, is especially rare. Reduced "TA" dinucleotide frequencies have been found in most prokaryotic genomes (Karlin et al. 2002).

In Halobacterium, the "CG" dinucleotide is much more frequent than the other dinucleotides consisting only of $\mathrm{G}$ and $C$ residues. As already noted by Karlin et al. (2002), an excess of "CG" is rather exceptional for prokaryotic genomes, which commonly are enriched for "GC." Indirectly, this "CG excess" facilitates gene selection and start codon assignment in Halobacterium to some extent, as it results in an excess of four trinucleotides, which correspond to arginine codons. Thus, translations of random stretches of DNA (spurious ORFs) are preferentially arginine-rich and thus highly alkaline, while halophilic proteins are known to be rich in aspartic acid and highly acidic: the pI value of $82 \%$ of the halobacterial proteins is between 3.5 and 5.5 (Tebbe et al. 2005).

Like spurious ORFs, $\mathrm{N}$-terminal gene extensions in front of the correct start codon tend to be highly alkaline, whereas the rest of the $\mathrm{N}$-terminal region of the protein tends to be acidic. In combination, this results in a large pI upshift in front of the correct start codon (see Fig. 6), which can help to assign it properly (Tebbe et al. 2005). In 

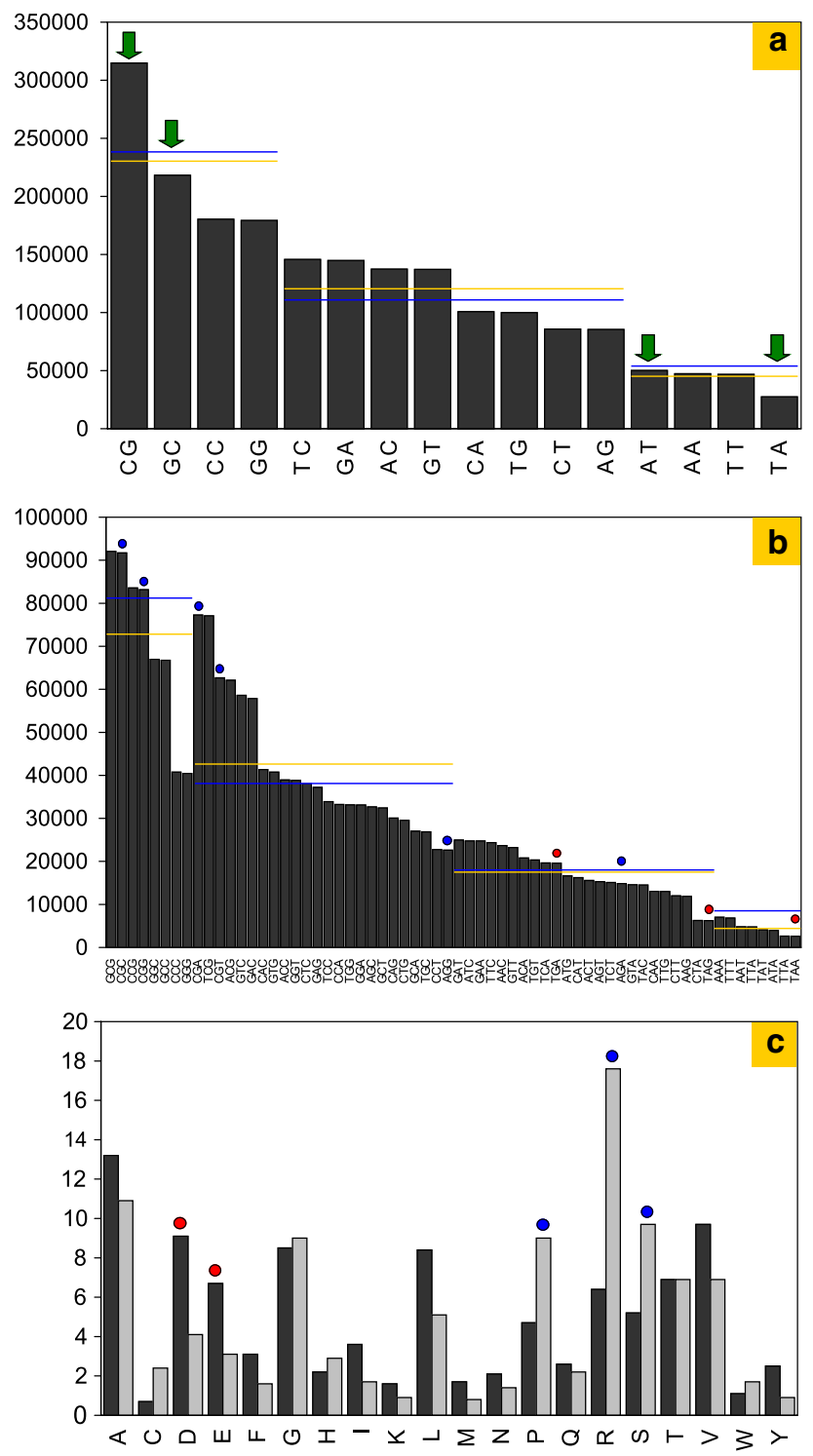

Fig. 5 Dinucleotide bias for Halobacterium salinarum. a Counts of dinucleotides in the Halobacterium salinarum chromosome. Dinucleotides are grouped according to the number of $\mathrm{G}$ or $\mathrm{C}$ residues. Within each group, each dinucleotide is adjacent to its reverse complement (e.g., TC and GA). The four palindromic dinucleotides are indicated by green arrows. For each group, the theoretically expected average (blue line) is compared with the average, which is actually observed (yellow line). b Same as (a) but showing the counts of trinucleotides. Red circles highlight stop codons and blue circles highlight trinucleotides that correspond to arginine codons. c The amino acid composition as computed from the protein-coding gene set (black) and from trinucleotide counts (gray). The over-representation of the acidic amino acids aspartate and to a lesser extent glutamate (red circles) in protein-coding genes contrasts with the over-representation of the basic amino acid arginine, prolines and serines (blue circles) in translations of random stretches of DNA. This is the basis for a strong $\mathrm{pI}$ difference between these two sets of ORFs

the HaloLex web interface, the indicative pI values are shown to assist the annotator in assigning the correct start codon (see "Section 1: overview of the HaloLex system").

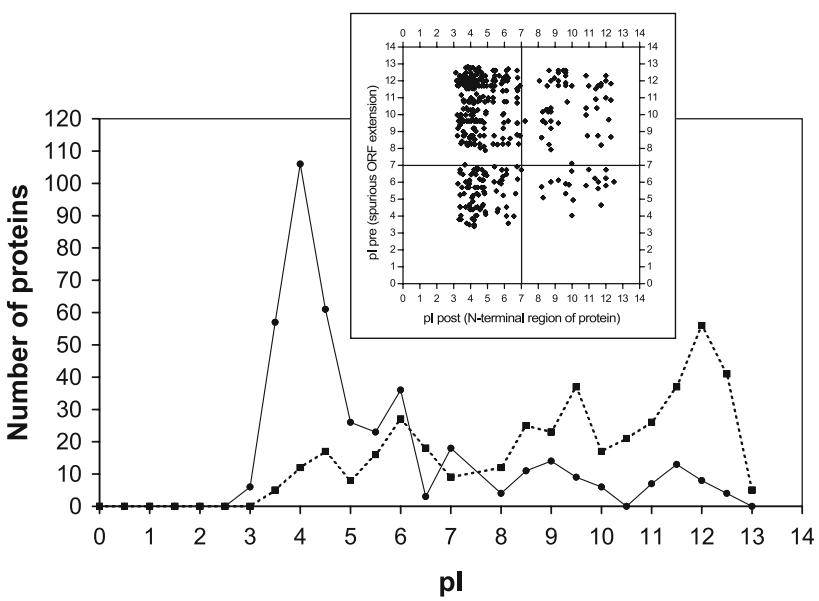

Fig. 6 pI shift around start codons. The distribution of $\mathrm{pI}$ values of the $20 \mathrm{~N}$-terminal residues excluding the initial Met (solid line) and the 20 residues of the spurious ORF extension (broken line) that precedes the start codon is plotted for Halobacterium. Transmembrane proteins and proteins with a signal sequence or twin-arginine export motif have been excluded from the analysis. The small inset shows the correlation of the $\mathrm{pI}$ value of the $\mathrm{N}$-terminal region of the protein (pI-post, plotted on the $x$-axis) and the $\mathrm{pI}$ value of the spurious ORF extension (pI_pre, plotted on the $y$-axis). The majority of the Nterminal regions are acidic, while a large fraction of the spurious extensions is highly alkaline

Integrated analysis of proteomic and genomic data

Gene selection and start codon assignment are greatly facilitated by experimental evidences, especially by proteome analysis. We have collected genome-scale proteomic data for Halobacterium salinarum (68\% of all proteins identified, Tebbe et al. 2005; Klein et al. 2005; Bisle et al. 2006; Falb et al. 2006; Aivaliotis et al. 2007) and for Natronomonas pharaonis (43\% of all proteins identified, Konstantinidis et al. 2007). This allowed to address and solve the two problems associated with gene prediction in GC-rich genomes, as an ORF is unambiguously confirmed as gene if the protein product is identified by a proteomic experiment. More than 100 orphans (ORFs that potentially code for proteins but do not have any homologs in the databases) could therewith be confirmed as genes. In many cases, initial gene predictions had to be corrected on the basis of proteomic data (see Tebbe et al. 2005).

No evidence for "ORF overprinting" (i.e., more than one gene is located on the same genomic sequence stretch, Keese and Gibbs 1992) was found in Halobacterium and Natronomonas, although throughout their chromosomes, more than one reading frame is open at a given genome location (cf. Fig. 2). Searching for protein identifications resulting from alternative overlapping reading frames, we did not find a single pair of identified overlapping proteins (see Aivaliotis et al. 2007). Therefore, we conclude that, if 
ORF overprinting occurs at all, it is a very rare event (Konstantinidis et al. 2007; Pfeiffer et al. 2008).

To address the problem of start codon assignment, we selected N-terminal peptides from the aforementioned set of proteomic data. In addition, we designed experiments in an attempt to specifically identify $\mathrm{N}$-terminal peptides (Aivaliotis et al. 2007). In total, N-termini from 606 proteins in $H$. salinarum and from 328 in $N$. pharaonis were identified (Falb et al. 2006; Aivaliotis et al. 2007). On the basis of these experimental data, the subsequent integrated analysis of proteomic and genomic data in the HaloLex system confirmed that commonly applied gene finders have a high error rate with respect to start codon selection (Falb et al. 2005; Aivaliotis et al. 2007). Major difficulties to assign correct start codons are also evident from the fact that several hundred start codon assignment discrepancies exist between Halobacterium salinarum strains R1 (Pfeiffer et al. 2008) and NRC-1 (Ng et al. 2000), although the DNA sequences are virtually identical. Whenever $\mathrm{N}$-terminal peptides could be identified by proteomics, they confirmed the start codon assignment for strain R1 (Pfeiffer et al. 2008).

A selection of additional results from our proteomic analysis illustrates the power of integrated analysis with the HaloLex system.

Our set of experimentally validated N-terminal peptides is among the largest in the prokaryotic world and allowed to unravel $\mathrm{N}$-terminal protein maturation in halophilic archaea, which consists of methionine cleavage and $\mathrm{N}$ terminal protein acetylation (Falb et al. 2006). N-terminal protein maturation critically depends on the penultimate residues (the one following the initiator-methionine). The set of proteins with N-terminally identified peptide contains 90 integral membrane proteins (again being one of the largest sets currently available). The data show that a major fraction of the integral membrane proteome is synthesized without a cleavable signal sequence and processed analogous to cytosolic proteins (Falb et al. 2006).

One focus of our group is on membrane proteins, which we have extensively analyzed by proteomics (Klein et al. 2005, Bisle et al. 2006). While identification of integral membrane proteins has become highly efficient, our data show that the identification of peptides that form the transmembrane domain is still in its infancy. Most of the integral membrane proteins are identified exclusively through loop peptides. Statistical analysis shows that this hampers protein modification-based quantitative proteomics of integral membrane proteins (Bisle et al. 2006).

Yet another issue concerning gene selection could be solved by experimental means. We were uncertain if our protein-coding gene set would show a major overprediction of small genes. Indicative of such an overprediction were two statistical results: (1) proteins smaller than $20 \mathrm{kDa}$ are severely underrepresented in the set of proteomically identified proteins (Tebbe et al. 2005; Klein et al. 2007); (2) although we had used gel systems that are able to separate proteins below $20 \mathrm{kDa}$, the number of $2 \mathrm{D}$ gel spots in this size range seems much smaller than expected from a theoretical 2D gel (Tebbe et al. 2005). Experimental analysis showed that the small proteins indeed exist, but have so far been missed due to technical problems in standard biochemical experiments. There is a severe washout of small proteins upon standard SDS gel handling procedures (Klein et al. 2007). Also, the low number of peptides upon tryptic digestion severely hampers proteomic identification. With improved experimental techniques, 380 proteins smaller than $20 \mathrm{kDa}$ could be identified (which increased the fraction of identified small proteins by a factor of six).

\section{Homology-based checking of ORF prediction}

Small protein-coding genes easily escape upon gene prediction. Therefore, we implemented a semiautomatic homology-based procedure to detect yet unannotated small genes. To this end, short protein sequences from closely related organisms are used for independent homology searches using blastP (protein vs. protein) and tblastN (protein vs. six-frame translation of the genome). Proteins with a higher score in tblastN as compared to blastP are selected for subsequent manual curation. Annotations of new genes, which are detected by this procedure, can be generated using a six-frame translator implemented in HaloLex. We applied this procedure to the published genome of Haloarcula marismortui, using proteins with up to 150 residues from $H$. salinarum strain R1, N. pharaonis, and $H$. walsbyi as a seed. This enabled us to detect 47 previously missed genes in Haloarcula (Table 1); among them, four were ribosomal proteins and 10 were small CPxCG-related zinc finger proteins, which are a prominent class of potential gene regulators found in all archaeal genomes (Tarasov et al. 2008).

In a similar way, sequence homology analysis allows to identify such genes, whose start codons were very likely incorrectly assigned. For this purpose, we analyze the results of a blastP search in closely related organisms. For each organism, the best homolog is used (provided the $e$ value is better than 1E-20). The alignment start position for query and hit is used to categorize the alignment. Alignments are considered to indicate a start codon misassignment if (1) the alignment starts very close to the $\mathrm{N}$-terminus for one sequence but far away for the other and (2) when the alignment starts at the initiator-methionine for one sequence and this methionine aligns with a potential start codon translation (Met or Val) in the other sequence. Candidates are further analyzed by manual inspection. Table 2 lists 337 genes from the published genome of Haloarcula marismortui, where we have reassigned the 
Table 1 Newly assigned genes in Haloarcula marismortui

\begin{tabular}{|c|c|c|c|c|c|}
\hline ORF & Length (aa) & Best homolog & Function & Seq id. $(\%)$ & Other homologs \\
\hline rrnAC0103_A & 75 & NP3662A & rib_prot S28.eR & 86 & OE2664F, HQ2884A \\
\hline rrnAC0208_A & 60 & NP0350A & $\mathrm{CHY}$ & 48 & - \\
\hline rrnAC0216_A & 126 & OE2874F & $\mathrm{CHY}$ & 42 & HQ1719A \\
\hline rrnAC0301_A & 80 & HQ2541A & Small $\mathrm{ZnF}$ & 45 & - \\
\hline rrnAC0669_A & 150 & NP0856A & $\mathrm{CHY}$ & 66 & HQ1219A, OE1540R \\
\hline rrnAC0678_A & 53 & NP0788A & Small $\mathrm{ZnF}$ & 73 & HQ1109A, OE1789R, HQ2748A, OE7210R \\
\hline rrnAC0696_A & 99 & NP0816A & Small $\mathrm{ZnF}$ & 47 & OE1556F \\
\hline rrnAC0797_A & 57 & HQ2892A & rib_prot L37.eR & 92 & OE3141R, NP4310A \\
\hline rrnAC0991_A & 48 & NP2998A & $\mathrm{CHY}$ & 79 & OE3047F \\
\hline rrnAC1044_A & 86 & HQ1848A & moaD family protein & 33 & NP2500A, NP5020A, NP3946A, OE3595R \\
\hline rrnAC1515_A & 66 & NP1736A & Small $\mathrm{ZnF}$ & 58 & HQ3220A, OE3365R \\
\hline rrnAC1588_A & 146 & OE5063R & IS200-type transposase & 72 & NP4630A, OE1439F, rrAC0815, OE4728F \\
\hline rrnAC1597_A & 61 & NP4882A & rib_prot S14 & 72 & OE3408F, HQ2828A, NP1768A \\
\hline rrnAC1603_A & 94 & NP4870A & RNAseP comp. 1 & 52 & OE3398F, HQ2834A \\
\hline rrnAC1676_A & 44 & NP4282A & $\mathrm{CHY}$ & 77 & NP2940A \\
\hline rrnAC1676_B & 122 & HQ1297A & $\mathrm{CHY}$ & 60 & - \\
\hline rrnAC1678_A & 100 & HQ1827A & $\mathrm{CHY}$ & 59 & - \\
\hline rrnAC1706_A & 141 & NP1764A & $\mathrm{CHY}$ & 52 & - \\
\hline rrnAC1831_A & 63 & NP1510A & $\mathrm{CHY}$ & 63 & HQ1704A, OE1775R \\
\hline rrnAC1867_A & 52 & HQ1176A & Small $\mathrm{ZnF}$ & 69 & OE1435R, NP5316A \\
\hline rrnAC1929_A & 212 & OE3249F & Cob cluster protein & 54 & NP5310A, HQ1412A, NP1896A \\
\hline rrnAC1936_A & 89 & NP3612A & $\mathrm{CHY}$ & 48 & - \\
\hline rrnAC1983_A & 231 & NP1896A & $\mathrm{CHY}$ & 43 & - \\
\hline rrnAC2105_A & 134 & NP0772A & $\mathrm{CHY}$ & 54 & HQ1375A, OE4661R \\
\hline rrnAC2167_A & 96 & NP4084A & $\mathrm{CHY}$ & 35 & HQ2323A \\
\hline rrnAC2212_A & 142 & HQ1071A & $\mathrm{CHY}$ & 38 & OE2090R \\
\hline rrnAC2268_A & 116 & NP2558A & Transcription regulator & 74 & OE2591R, NP3596A, rrnAC3399, HQ1949A \\
\hline rrnAC2270_A & 59 & HQ1365A & Small $\mathrm{ZnF}$ & 68 & NP0928A, OE4676F \\
\hline rrnAC2286_A & 84 & NP5336A & $\mathrm{CHY}$ & 43 & - \\
\hline rrnAC2448_A & 54 & NP5086A & $\mathrm{CHY}$ & 51 & - \\
\hline rrnAC2530_A & 130 & HQ2261A & $\mathrm{CHY}$ & 52 & - \\
\hline rrnAC2569_A & 49 & HQ3677A & Small $\mathrm{ZnF}$ & 74 & NP0778A, OE4167C1R, HQ2748A, OE7210R \\
\hline rrnAC2574_A & 52 & NP0788A & Small $\mathrm{ZnF}$ & 92 & OE1789R, HQ1109A, HQ2748A \\
\hline rrnAC2592_A & 98 & HQ1034A & $\mathrm{CHY}$ & 79 & NP1820A \\
\hline rrnAC2764_A & 111 & NP5102A & $\mathrm{CHY}$ & 59 & HQ3659A, OE4054F \\
\hline rrnAC2791_A & 115 & NP0196A & $\mathrm{CHY}$ & 70 & HQ3647A, OE3914R \\
\hline rrnAC2834_A & 129 & OE4148F & $\mathrm{CHY}$ & 31 & HQ3411A \\
\hline rrnAC2897_A & 73 & OE4475R & Small $\mathrm{ZnF}$ & 58 & HQ3437A, NP0708A \\
\hline rrnAC2982_A & 137 & HQ2813A & $\mathrm{CHY}$ & 42 & HQ1789A, HQ2547A, pNG7092, NP1808A \\
\hline rrnAC3115_A & 57 & NP0186A & rib_prot HL32 & 75 & HQ3421A \\
\hline rrnB0024_A & 139 & OE6004F & Small $\mathrm{ZnF}$ & 64 & NP6252A, HQ1149A \\
\hline rrnB0146_A & 118 & OE1549F & $\mathrm{CHY}$ & 54 & NP1698A, HQ1429A, OE3894R \\
\hline rrnB0177_A & 89 & HQ2065A & $\mathrm{CHY}$ & 50 & - \\
\hline pNG3034_A & 47 & NP4282A & $\mathrm{CHY}$ & 80 & NP2940A \\
\hline pNG6117_A & 85 & OE6052R & $\mathrm{CHY}$ & 59 & - \\
\hline pNG6164_A & 115 & OE6242R & $\mathrm{CHY}$ & 79 & - \\
\hline pNG6170_A & 53 & NP0788A & $\mathrm{CHY}$ & 75 & OE1789R, HQ1109A, HQ2748A, OE7210R \\
\hline
\end{tabular}

Using tblastN, previously unannotated genes were detected and realized by the manual curation options within HaloLex. For each newly assigned gene, its code, length, the best homolog (with a brief function indication and percentage of sequence identity) and other homologous genes are given. Codes are systematically assigned using the number of the upstream ORF and a letter attached with an intervening underscore (commonly _A). Function assignment abbreviations: $C H Y$ conserved hypothetical protein, rib_prot ribosomal protein, small ZnF small CPxCG-related zinc finger protein (Tarasov et al. 2008) 


\begin{abstract}
VNG2591C mrlvqvtvptgkrdavlaalddegvdyvvtpetasteytavvhfplptaavsdvldalqdvglsqdaytvvvdaetvvsr OE4634F MRLVQVTVPTGKRDAVLAALDDEGVDYVVTPETASTEYTAVVHFPLPTAAVSDVLDALQDVGLSQDAYTVVVDAETVVSR HO3141A MRFVQVLVPAGTRDAVVEVITDENIEYAITDETGTDEYEAIITFPLPTAAVEPVLDQLRTVGIDTDATTVVLEAETVVSD NP0578A MRLVQVTIPAGKREAVLRVLDEEGIDYVVTDETSGREYTAVAYFPLPTSAVEPILEQLRDVGLEREAYTVVVSAETVVSK rrnAC2377 mrlvqliliptgkrdavlgvlteegidyvltdetsgreftavvtfpvptnalepvlealrdvginddgytvvvdantviss

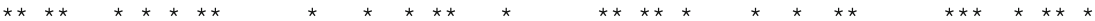

VNG2591C rfdeldarysadsdaadd--hiareelvaraadlapnrvtyavltlvstiiatagllldspatvvgsMVIAPLLGPAMSA OE4634F RFDELDARYSADSDAADD--HIAREELVARAADLAPNRVTYAVLTLVSTI IATAGLLLDSPATVVGSMVIAPLLGPAMSA HO3141A KFDELDEAYSNDSDDTTDGDRIARDELLARANELAPG IGPF ILMT IVSAIVATAGLLLDSPAVVVGSMVIAPLIGPAMST NP0578A RFDDLKDSYAEKEESEE---RIARQEIEARAEELAAS IPTYVVMTIVSAVIATAGLLLDSPATVVGSMVIAPLIGPAMTT

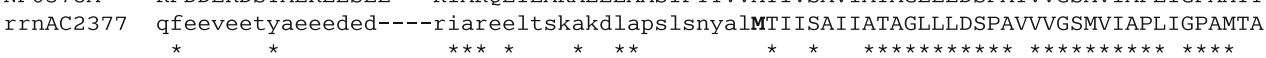

VNG2591C SVGTVVDDDDLFARGIRLQVVGVALAVVGAAAFAFLVKTTHLVP-PGLDVLSLSEVRERLRPDFLSLVVALGSGVAGVYS OE4634F SVGTVVDDDDLFARGIRLQVVGVALAVVGAAAFAFLVKTTHLVP-PGLDVLSLSEVRERLRPDFLSLVVALGSGVAGVYS HQ3141A SVGTVVDDTSLVARGVKLOILGGVLAIVSAAGFAFMLRTVOIVPLSAVEVFEIGEVSORLAPDVLSLVIALGAGAAGAVS NP0578A AVGSVIDDAELFQRGVSLQVVGIVLAVAAATVFAVFVQVMNLVP-PGLDPLSLAEVEERLSPNFLSLAVAIGAGIAGAVS rrnAC2377 NVGTVVDDNEMFARGVKLQAVGLGLAVASATAFALLVRYANVIP-PLADVTAVGQIRERVAPDFLSLIVALGAGAAGVVS

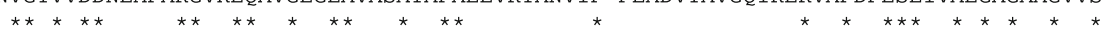

Fig. 7 Homology-based start codon checking for the detection of ORFs, which are too short. A sequence alignment of four homologous proteins of $H$. salinarum (strains R1 and NRC-1), $N$. pharaonis, $H$. walsbyi and $H$. marismortui is shown. Codes starting with $\mathrm{OE}$ are from $H$. salinarum strain R1, those with VNG from strain NRC-1, NP from $N$. pharaonis, HQ from $H$. walsbyi and those starting with rrnAC from H. marismortui. Uppercase letters indicate the protein sequence as obtained from the current database, the first methionine being bold. Lowercase letters indicate additional residues obtained by our correction of the start codon assignment. Residues conserved in all sequences are indicated by asterisks

$\begin{array}{lr}\text { VNG2422C } & \text { MAYSGPPKHHAQPSGPHHRPQRSHAPTHYATP } \\ \text { OE4429F } & \text { maysgppkhaqpsgphhrpqrshapthyatp } \\ \text { HQ3640A } & - \\ \text { NP0462A } & \text { mtpalsipylcrshqkgldsaglgpncqpldvlnrrfaadavlvgdglrgr } \\ \text { rrnAC2722 } & \text { MTQNASGFIPGSTTGS }\end{array}$

VNG2422C ${ }^{33}$ MIAVLADTHSDTDHALTGHARQAVADADAVVHAGDFTTESSLDAFHDAATRLHAVHGNADSPAVRDRLPP OE4429F ${ }^{1}$ MIAVLADTHSDTDHALTGHARQAVADADAVVHAGDFTTESSLDAFHDAATRLHAVHGNADSPAVRDRLPP HO3640A ${ }^{1}$ MLTVISDTHSTDNHOLSGOTYEAVONAEMVAHAGDFMCESVLDALOREATOLVGVAGNNDDTGIRERLPT NP0462A ${ }^{1}$ MLAVLSDTHGRDSPRLSGRTADAVAEASRVVHAGDFMTEAVLDAFEERGP-LAAVYGNNATAAVRERLPA rrnAC2722 ${ }^{17}$ MLTAISDTHGTDNHRLTGRTLDAVREADHVLHAGDFMTEQVLDAIDAESDELTGVVGNNDRPAVRARLSD

Fig. 8 Homology-based start codon checking for the detection of ORFs, which are too long. A sequence alignment of four homologous proteins of $H$. salinarum (strains R1 and NRC-1), N. pharaonis, $H$. walsbyi and $H$. marismortui is shown. Codes starting with $\mathrm{OE}$ are from $H$. salinarum strain R1, those with VNG from strain NRC-1, NP from $N$. pharaonis, HQ from $H$. walsbyi and those starting with rrnAC from $H$. marismortui. The protein sequences are highly homologous. Residues conserved in all sequences are indicated by asterisks (lower

start codon (196 genes are shortened and 141 extended). We briefly discuss two example cases by showing the corresponding multiple sequence alignments (Figs. 7, 8).

Figure 7 shows a gene, which needs to be extended in Haloarcula marismortui (and also in $H$. salinarum strain NRC-1). Met-1 of rrnAC2377 aligns with Met-121 of NP0578A. Using the longer sequence (here NP0578) for tblastN shows that the homologous region extends beyond the assigned start codon (lowercase sequence letters for rrnAC2377). VNG2591C can also be extended to match $\mathrm{OE} 4634 \mathrm{~F}$, as the genome sequences of strains $\mathrm{R} 1$ and NRC-1 are identical in this region (also indicated by lowercase sequence letters).

Figure 8 shows an example of a gene, which needs to be shortened in Haloarcula marismortui (and also in

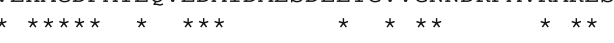

alignment block). Spurious $\mathrm{N}$-terminal sequence extensions are possible in three of the four species, but are considered to be incorrect as they are not homologous to each other (upper alignment block). Uppercase letters indicate the protein sequence as obtained from the current database, the first methionine being bold. The position of the probable initiator methionine in the current database sequence is indicated. Lowercase letters indicate gene extensions, which are possible but are considered spurious

H. salinarum strain NRC-1). The methionine at position 17 in the rrnAC2722 sequence aligns with the methionine at position 1 of NP0462A. Using the longer sequence (here rrnAC2722) for tblastN does not result in an extension of the homologous region as compared to the shorter sequence (NP0462A), which indicates that the extension may be spurious. Spurious ORF extensions are possible in three of the four halophiles, but they are not homologous to each other.

It should be stressed that the homology-based procedures described above are not suitable for performing automatic, unsupervised gene predictions. They rather serve to preselect candidates with probable gene prediction errors, which then need to be manually inspected. The HaloLex system is well suited to support such manual 
Table 2 Genes with corrected start codon assignments in Haloarcula marismortui

\begin{tabular}{|c|c|c|c|c|}
\hline ORF & $\begin{array}{l}\text { Corrected } \\
\text { length (aa) }\end{array}$ & $\begin{array}{l}\text { Original } \\
\text { length (aa) }\end{array}$ & $\begin{array}{l}\text { Direction } \\
\text { of change }\end{array}$ & Homologous ORFs \\
\hline rrnAC0004 & 1,551 & 1,356 & Extended & NP4364A, OE3175F, HQ3018A \\
\hline rrnAC0005 & 624 & 360 & Extended & OE2052F, NP3952A \\
\hline $\operatorname{rrnAC0012}$ & 957 & 1,083 & Shortened & HQ1987A, NP4816A \\
\hline rrnAC0041 & 279 & 339 & Shortened & NP3812A, HQ1503A, OE2950R \\
\hline $\operatorname{rrnAC0053}$ & 864 & 717 & Extended & NP2042A, HQ3014A \\
\hline $\operatorname{rrnAC0080}$ & 1,347 & 1,383 & Shortened & NP3698A, OE2648F, HQ2890A \\
\hline rrnAC0083 & 936 & 654 & Extended & NP1382A, HQ2430A \\
\hline rrnAC0101 & 2,238 & 2,328 & Shortened & OE2656R, NP3690A \\
\hline $\operatorname{rrnAC} 0115$ & 774 & 909 & Shortened & NP4142A, OE2472F \\
\hline $\operatorname{rrnAC0137}$ & 882 & 993 & Shortened & OE2860R, NP3116A, rrnAC1236 \\
\hline rrnAC0145 & 1,125 & 843 & Extended & OE4359F, HQ1341A \\
\hline $\operatorname{rrnAC} 0171$ & 984 & 1,080 & Shortened & OE1599F \\
\hline $\operatorname{rrnAC} 0178$ & 1,812 & 1,725 & Extended & OE1613R \\
\hline $\operatorname{rrnAC0181}$ & 1,866 & 1,413 & Extended & NP4200A, OE3010F, HQ2528A \\
\hline rrnAC0198 & 1,236 & 1,305 & Shortened & NP1646A \\
\hline rrnAC0199 & 480 & 537 & Shortened & NP1296A \\
\hline rrnAC0213 & 951 & 726 & Extended & HQ1261A \\
\hline $\operatorname{rrnAC} 0215$ & 723 & 522 & Extended & NP0356A, HQ2398A, OE1636F \\
\hline rrnAC0239 & 999 & 1,110 & Shortened & NP1902A, OE2918F \\
\hline $\operatorname{rrnAC} 0240$ & 333 & 372 & Shortened & OE3652F, HQ2230A \\
\hline $\operatorname{rrnAC} 0249$ & 879 & 1,017 & Shortened & OE3606R, NP3184A \\
\hline $\operatorname{rrnAC0261}$ & 267 & 300 & Shortened & HQ2898A, NP3686A, OE3683R \\
\hline $\operatorname{rrnAC} 0280$ & 333 & 381 & Shortened & NP2580A, HQ2722A \\
\hline $\operatorname{rrnAC} 0284$ & 447 & 225 & Extended & NP2596A, HQ2724A \\
\hline rrnAC0304 & 1,104 & 1,155 & Shortened & OE2360R, NP5226A \\
\hline rrnAC0305 & 957 & 1,011 & Shortened & OE2551F, NP3722A \\
\hline $\operatorname{rrnAC0322}$ & 1,176 & 1,203 & Shortened & NP3076A, OE2763F \\
\hline $\operatorname{rrnAC0324}$ & 411 & 153 & Extended & NP3362A, OE4451F \\
\hline $\operatorname{rrnAC0329}$ & 1,245 & 1,035 & Extended & NP2206A, HQ2700A \\
\hline $\operatorname{rrnAC0374}$ & 414 & 324 & Extended & NP2642A, OE2237F, HQ2615A \\
\hline rrnAC0394 & 1,008 & 1,056 & Shortened & NP1082A, OE4339R, HQ3696A \\
\hline $\operatorname{rrnAC} 0426$ & 1,386 & 1,464 & Shortened & pNG7203, NP0964A, HQ3464A \\
\hline $\operatorname{rrnAC} 0430$ & 936 & 975 & Shortened & HQ2692A, NP1916A, OE2547R \\
\hline $\operatorname{rrnAC0436}$ & 1,530 & 1,605 & Shortened & OE2288F, NP2702A, HQ2668A \\
\hline $\operatorname{rrnAC0481}$ & 1,002 & 894 & Extended & NP2798A \\
\hline rrnAC0494 & 249 & 306 & Shortened & NP4192A, OE1860F, HQ1556A \\
\hline rrnAC0497 & 708 & 471 & Extended & HQ1562A, OE1794R \\
\hline rrnAC0505 & 1,710 & 1,485 & Extended & OE3490R, NP1742A, HQ3347A \\
\hline rrnAC0506 & 1,527 & 1,575 & Shortened & NP3956A, OE2049R \\
\hline $\operatorname{rrnAC0536}$ & 573 & 606 & Shortened & NP2906A, HQ2751A \\
\hline $\operatorname{rrnAC0546}$ & 1,791 & 1,833 & Shortened & HQ1573A, OE1495R, NP1746A \\
\hline rrnAC0568 & 699 & 435 & Extended & HQ1615A, rrnAC3127, NP1996A \\
\hline $\operatorname{rrnAC0572}$ & 804 & 855 & Shortened & HQ3669A, rrnAC2557, NP0792A, OE3115F \\
\hline rrnAC0589 & 657 & 846 & Shortened & $\operatorname{rrnAC} 2321, \mathrm{OE} 8048 \mathrm{~F}$ \\
\hline $\operatorname{rrnAC0617}$ & 1,335 & 1,413 & Shortened & NP0212A, HQ2634A, OE8010R \\
\hline rrnAC0619 & 1,272 & 963 & Extended & HQ3141A, OE4634F, NP0578A \\
\hline rrnAC0620 & 1,566 & 1,431 & Extended & NP4066A, HQ2635A, OE7174R \\
\hline $\operatorname{rrnAC0628}$ & 912 & 957 & Shortened & NP4072A, HQ2637A, OE1748R \\
\hline rrnAC0629 & 738 & 774 & Shortened & NP4074A, HQ2638A, OE1752F \\
\hline
\end{tabular}


Table 2 continued

\begin{tabular}{|c|c|c|c|c|}
\hline ORF & $\begin{array}{l}\text { Corrected } \\
\text { length (aa) }\end{array}$ & $\begin{array}{l}\text { Original } \\
\text { length (aa) }\end{array}$ & $\begin{array}{l}\text { Direction } \\
\text { of change }\end{array}$ & Homologous ORFs \\
\hline $\operatorname{rrnAC0631}$ & 1,122 & 642 & Extended & NP0380A, OE1582R, HQ1531A \\
\hline $\operatorname{rrnAC0633}$ & 969 & 1,008 & Shortened & NP0384A, OE1578F, HQ1670A \\
\hline $\operatorname{rrnAC0638}$ & 474 & 606 & Shortened & HQ3692A, NP2228A \\
\hline $\operatorname{rrnAC0651}$ & 1,191 & 1,059 & Extended & OE4393R, NP0888A \\
\hline $\operatorname{rrnAC0655}$ & 603 & 657 & Shortened & NP1390A, HQ1666A \\
\hline $\operatorname{rrnAC0660}$ & 333 & 417 & Shortened & NP4090A, HQ2743A, OE1651F \\
\hline $\operatorname{rrnAC0663}$ & 1,029 & 240 & Extended & NP0372A, OE1646R, HQ2392A \\
\hline rrnAC0666 & 708 & 582 & Extended & NP0100A, HQ3478A, OE1004F \\
\hline rrnAC0674 & 810 & 945 & Shortened & rrnAC0848, OE7042R, rrnAC2044, HQ2141A, NP6028A \\
\hline rrnAC0687 & 1,269 & 1,347 & Shortened & OE4207F \\
\hline rrnAC0696 & 762 & 900 & Shortened & NP0818A, OE1554R \\
\hline $\operatorname{rrnAC0717}$ & 636 & 699 & Shortened & NP1230A, OE1713F, HQ1537A \\
\hline $\operatorname{rrnAC0721}$ & 1,371 & 1,395 & Shortened & OE3467R, HQ1298A \\
\hline $\operatorname{rrnAC0753}$ & 879 & 936 & Shortened & OE2785R, HQ2762A \\
\hline $\operatorname{rrnAC0777}$ & 903 & 492 & Extended & HQ2440A \\
\hline $\operatorname{rrnAC0779}$ & 861 & 915 & Shortened & OE2138F, NP1596A \\
\hline $\operatorname{rrnAC0801}$ & 951 & 1,071 & Shortened & NP4302A, OE3145F, HQ2933A \\
\hline $\operatorname{rrnAC0825}$ & 1,284 & 1,383 & Shortened & NP4134A, HQ2196A \\
\hline $\operatorname{rrnAC0833}$ & 795 & 858 & Shortened & NP1462A, OE1641R, HQ2394A \\
\hline $\operatorname{rrnAC0838}$ & 1,779 & 1,827 & Shortened & NP2726A, HQ1873A, OE2653R \\
\hline $\operatorname{rrnAC} 0841$ & 954 & 1,062 & Shortened & NP2730A, OE2561R, HQ1874A \\
\hline rrnAC0843 & 1,524 & 1,587 & Shortened & NP2738A, OE2555R, HQ2402A \\
\hline $\operatorname{rrnAC0852}$ & 1,017 & 966 & Extended & OE3343R \\
\hline $\operatorname{rrnAC0875}$ & 543 & 405 & Extended & OE3121R, HQ2788A \\
\hline rrnAC0878 & 810 & 783 & Extended & OE3119R, NP4334A \\
\hline $\operatorname{rrnAC0883}$ & 1,116 & 504 & Extended & NP4236A \\
\hline rrnAC0896 & 1,152 & 1,293 & Shortened & HQ1590A, OE2358F, NP3650A \\
\hline $\operatorname{rrnAC0917}$ & 1,065 & 1,140 & Shortened & HQ1663A, OE1669F \\
\hline rrnAC0925 & 990 & 1,035 & Shortened & NP2796A, OE2451R \\
\hline $\operatorname{rrnAC0934}$ & 423 & 471 & Shortened & NP2710A, OE2005F, HQ2301A \\
\hline $\operatorname{rrnAC0942}$ & 1,611 & 1,416 & Extended & OE3436R \\
\hline rrnAC0944 & 1,302 & 1,350 & Shortened & HQ1663A, OE1669F \\
\hline $\operatorname{rrnAC0956}$ & 462 & 537 & Shortened & HQ1497A, OE2934R \\
\hline $\operatorname{rrnAC} 1042$ & 1,806 & 1,851 & Shortened & rrnAC1570, HQ3533A \\
\hline $\operatorname{rrnAC} 1083$ & 1,965 & 2,010 & Shortened & NP4322A, OE2871F \\
\hline rrnAC1106 & 519 & 420 & Extended & NP4198A, OE2985F, HQ2561A \\
\hline $\operatorname{rrnAC} 1107$ & 1,476 & 1,176 & Extended & NP4904A, HQ1686A \\
\hline $\operatorname{rrnAC} 1115$ & 270 & 324 & Shortened & NP4036A, OE2903R, HQ2458A \\
\hline $\operatorname{rrnAC} 1138$ & 849 & 507 & Extended & OE2020F, NP1592A \\
\hline $\operatorname{rrnAC} 1169$ & 1,311 & 867 & Extended & NP3742A, OE2827R, HQ2339A \\
\hline $\operatorname{rrnAC} 1218$ & 1,794 & 1,821 & Shortened & HQ1754A \\
\hline $\operatorname{rrnAC} 1220$ & 663 & 606 & Extended & HQ1752A \\
\hline $\operatorname{rrnAC} 1261$ & 1,155 & 1,182 & Shortened & NP4050A, HQ2389A \\
\hline $\operatorname{rrnAC} 1263$ & 798 & 894 & Shortened & OE2913R, NP3970A \\
\hline $\operatorname{rrnAC} 1281$ & 2,529 & 2,592 & Shortened & OE2573F, NP1526A \\
\hline $\operatorname{rrnAC} 1299$ & 1,881 & 1,704 & Extended & OE1143R, HQ3344A, NP1442A \\
\hline $\operatorname{rrnAC} 1308$ & 339 & 399 & Shortened & NP2066A, HQ1665A, OE1673F \\
\hline $\operatorname{rrnAC} 1336$ & 399 & 426 & Shortened & NP4972A \\
\hline $\operatorname{rrnAC} 1341$ & 1,683 & 1,800 & Shortened & NP0164A \\
\hline
\end{tabular}


Table 2 continued

\begin{tabular}{|c|c|c|c|c|}
\hline ORF & $\begin{array}{l}\text { Corrected } \\
\text { length (aa) }\end{array}$ & $\begin{array}{l}\text { Original } \\
\text { length (aa) }\end{array}$ & $\begin{array}{l}\text { Direction } \\
\text { of change }\end{array}$ & Homologous ORFs \\
\hline $\operatorname{rrnAC} 1350$ & 1,050 & 1,191 & Shortened & NP3216A, OE1906R, HQ2500A \\
\hline $\operatorname{rrnAC} 1361$ & 687 & 498 & Extended & OE2276F, NP2980A, HQ1692A \\
\hline $\operatorname{rrnAC} 1365$ & 1,026 & 1,161 & Shortened & $\operatorname{rrnAC} 0576$ \\
\hline $\operatorname{rrnAC} 1377$ & 582 & 777 & Shortened & rrnAC0508, NP3954A \\
\hline $\operatorname{rrnAC} 1383$ & 210 & 342 & Shortened & NP1548A \\
\hline $\operatorname{rrnAC} 1395$ & 849 & 1,029 & Shortened & NP4160A \\
\hline $\operatorname{rrnAC} 1438$ & 429 & 351 & Extended & NP3220A, OE2139R, HQ1579A \\
\hline rrnAC1443 & 1,275 & 1,398 & Shortened & NP3228A, HQ1584A, OE2149R \\
\hline rrnAC1444 & 1,623 & 1,746 & Shortened & HQ1934A, HQ2096A, pNG7256 \\
\hline $\operatorname{rrnAC} 1447$ & 414 & 534 & Shortened & NP2292A, HQ1637A, OE1953F \\
\hline $\operatorname{rrnAC} 1454$ & 303 & 207 & Extended & OE1963F, HQ1645A, NP2308A \\
\hline $\operatorname{rrnAC} 1477$ & 1,047 & 1,251 & Shortened & OE2014F, HQ2353A \\
\hline $\operatorname{rrnAC} 1497$ & 1,431 & 1,707 & Shortened & NP4594A, OE3274R \\
\hline $\operatorname{rrnAC} 1500$ & 1,092 & 1,155 & Shortened & OE3278R, NP4774A \\
\hline $\operatorname{rrnAC} 1504$ & 846 & 528 & Extended & NP4780A, HQ2866A, OE3286F \\
\hline $\operatorname{rrnAC} 1516$ & 189 & 489 & Shortened & OE3330F \\
\hline $\operatorname{rrnAC} 1530$ & 765 & 459 & Extended & NP1786A, HQ3174A \\
\hline $\operatorname{rrnAC} 1532$ & 711 & 579 & Extended & NP1788A, OE3352R, HQ3173A \\
\hline $\operatorname{rrnAC} 1536$ & 1,332 & 1,395 & Shortened & HQ1685A, NP4902A, OE5298F \\
\hline $\operatorname{rrnAC} 1542$ & 1,416 & 1,644 & Shortened & OE1133F \\
\hline $\operatorname{rrnAC} 1567$ & 291 & 474 & Shortened & HQ2131A \\
\hline $\operatorname{rrnAC} 1588$ & 1,308 & 882 & Extended & OE5062R \\
\hline $\operatorname{rrnAC} 1621$ & 570 & 459 & Extended & HQ2801A, OE3367F \\
\hline $\operatorname{rrnAC} 1626$ & 2,532 & 2,808 & Shortened & rrnAC2044, rrnAC0848, OE7042R, HQ2141A \\
\hline $\operatorname{rrnAC} 1628$ & 366 & 216 & Extended & OE3324R, HQ2783A, NP3352A \\
\hline $\operatorname{rrnAC} 1630$ & 591 & 411 & Extended & OE2334R, NP0028A \\
\hline $\operatorname{rrnAC} 1638$ & 975 & 1,107 & Shortened & NP1214A \\
\hline $\operatorname{rrnAC} 1647$ & 402 & 222 & Extended & NP1834A \\
\hline $\operatorname{rrnAC} 1655$ & 750 & 675 & Extended & NP1082A, OE4339R, HQ3696A \\
\hline $\operatorname{rrnAC} 1665$ & 642 & 735 & Shortened & NP2884A \\
\hline $\operatorname{rrnAC} 1669$ & 279 & 351 & Shortened & OE1371R, HQ1283A, NP5232A \\
\hline $\operatorname{rrnAC} 1680$ & 390 & 429 & Shortened & NP0612A, OE1371R, HQ1286A \\
\hline $\operatorname{rrnAC} 1690$ & 1,545 & 1,509 & Extended & NP0624A, HQ1292A \\
\hline $\operatorname{rrnAC} 1702$ & 1,719 & 1,218 & Extended & NP1742A, OE3490R, HQ3347A \\
\hline $\operatorname{rrnAC} 1708$ & 1,347 & 1,089 & Extended & NP4502A, HQ3336A, OE3496R \\
\hline $\operatorname{rrnAC} 1718$ & 1,359 & 1,065 & Extended & NP4542A, OE3506F, HQ3330A \\
\hline rrnAC1726 & 1,527 & 1,485 & Extended & HQ3326A, OE3511F, NP4534A \\
\hline rrnAC1743 & 549 & 486 & Extended & HQ1673A, NP5358A, rrnAC3526 \\
\hline $\operatorname{rrnAC} 1764$ & 924 & 978 & Shortened & rrnAC1777, NP5168A, OE1385F, HQ1277A \\
\hline $\operatorname{rrnAC} 1774$ & 339 & 411 & Shortened & HQ1279A, OE1379R \\
\hline $\operatorname{rrnAC} 1776$ & 588 & 633 & Shortened & NP5166A, OE1384F, HQ1278A \\
\hline $\operatorname{rrnAC} 1779$ & 651 & 516 & Extended & NP5170A \\
\hline $\operatorname{rrnAC} 1782$ & 933 & 963 & Shortened & HQ1276A, NP5174A, OE4651F \\
\hline $\operatorname{rrnAC} 1797$ & 858 & 903 & Shortened & NP4932A, OE3445F, rrnAC0317 \\
\hline $\operatorname{rrnAC} 1809$ & 735 & 444 & Extended & OE1445R, NP1134A \\
\hline $\operatorname{rrnAC} 1812$ & 705 & 525 & Extended & OE1451F, HQ1168A, NP1178A \\
\hline $\operatorname{rrnAC} 1822$ & 717 & 666 & Extended & NP1636A, OE1793F, HQ1712A \\
\hline $\operatorname{rrnAC} 1826$ & 522 & 237 & Extended & NP1498A, HQ1709A, OE1785F \\
\hline $\operatorname{rrnAC} 1840$ & 2,676 & 2,727 & Shortened & NP1516A, OE1770F, HQ1701A \\
\hline
\end{tabular}


Table 2 continued

\begin{tabular}{|c|c|c|c|c|}
\hline ORF & $\begin{array}{l}\text { Corrected } \\
\text { length (aa) }\end{array}$ & $\begin{array}{l}\text { Original } \\
\text { length (aa) }\end{array}$ & $\begin{array}{l}\text { Direction } \\
\text { of change }\end{array}$ & Homologous ORFs \\
\hline $\operatorname{rrnAC} 1849$ & 1,746 & 1,083 & Extended & HQ1189A, NP5206A \\
\hline $\operatorname{rrnAC} 1853$ & 3,003 & 2,616 & Extended & NP5214A, HQ1185A \\
\hline $\operatorname{rrnAC} 1855$ & 891 & 330 & Extended & NP5218A, OE1417F, HQ1183A \\
\hline $\operatorname{rrnAC} 1867$ & 522 & 756 & Shortened & HQ1177A, OE1434R, NP5318A \\
\hline $\operatorname{rrnAC} 1870$ & 1,455 & 1,209 & Extended & NP4702A, OE3960F \\
\hline $\operatorname{rrnAC} 1880$ & 1,239 & 1,284 & Shortened & NP0438A, OE3971R, rrnAC3166 \\
\hline $\operatorname{rrnAC} 1905$ & 429 & 279 & Extended & NP4526A, pNG6069 \\
\hline rrnAC1930 & 924 & 366 & Extended & OE3253F, NP5308A, HQ1411A \\
\hline $\operatorname{rrnAC} 1931$ & 804 & 504 & Extended & HQ1410A, NP5306A, OE3255F \\
\hline rrnAC1950 & 1,893 & 1,680 & Extended & NP0158A, HQ1329A \\
\hline rrnAC1957 & 1,491 & 1,671 & Shortened & HQ2578A \\
\hline rrnAC1979 & 795 & 591 & Extended & NP1462A, OE1641R \\
\hline $\operatorname{rrnAC} 1983$ & 1,218 & 1,755 & Shortened & NP1754A, OE2013R \\
\hline $\operatorname{rrnAC} 1992$ & 738 & 591 & Extended & NP1470A \\
\hline $\operatorname{rrnAC} 2014$ & 792 & 747 & Extended & NP5122A, OE1306F, HQ1416A \\
\hline $\operatorname{rrnAC} 2085$ & 360 & 522 & Shortened & NP0342A, OE4713R, HQ3071A \\
\hline rrnAC2098 & 1,878 & 1,905 & Shortened & NP0198A, OE4671R, HQ1369A \\
\hline $\operatorname{rrnAC} 2105$ & 522 & 1,014 & Shortened & NP0774A, OE4663F, HQ1347A \\
\hline $\operatorname{rrnAC} 2127$ & 1,005 & 711 & Extended & NP0962A \\
\hline $\operatorname{rrnAC} 2129$ & 864 & 894 & Shortened & OE4355R, NP3186A \\
\hline $\operatorname{rrnAC} 2158$ & 1,974 & 2,034 & Shortened & NP0404A, OE4613F, HQ3117A \\
\hline rrnAC2159 & 462 & 609 & Shortened & NP0954A, HQ3116A, OE4610R \\
\hline $\operatorname{rrnAC} 2181$ & 1,098 & 1,227 & Shortened & NP1140A, OE4571R, HQ1074A \\
\hline $\operatorname{rrnAC} 2221$ & 435 & 579 & Shortened & OE4541F, NP1718A, HQ1065A, rrnAC2455 \\
\hline $\operatorname{rrnAC} 2223$ & 372 & 387 & Shortened & NP1710A, OE4544R, HQ1063A \\
\hline $\operatorname{rrnAC} 2245$ & 1,137 & 870 & Extended & OE4034R, HQ3066A, NP0030A \\
\hline $\operatorname{rrnAC} 2247$ & 1,296 & 1,443 & Shortened & NP1050A \\
\hline $\operatorname{rrnAC} 2258$ & 624 & 435 & Extended & NP0018A \\
\hline $\operatorname{rrnAC} 2261$ & 954 & 1,026 & Shortened & OE1151R, NP0014A, HQ1359A \\
\hline $\operatorname{rrnAC} 2278$ & 528 & 600 & Shortened & NP3368A, HQ2565A, rrnAC0868, OE2992R \\
\hline $\operatorname{rrnAC} 2284$ & 1,038 & 993 & Extended & NP5368A, OE2438R \\
\hline $\operatorname{rrnAC} 2352$ & 1,167 & 1,188 & Shortened & pNG7026, OE5170F, HQ1989A \\
\hline $\operatorname{rrnAC} 2356$ & 999 & 1,251 & Shortened & NP5048A, HQ1275A, OE4196R \\
\hline $\operatorname{rrnAC} 2359$ & 432 & 180 & Extended & NP4806A, rrnAC0738, OE3162F, HQ2346A \\
\hline $\operatorname{rrnAC} 2377$ & 1,281 & 924 & Extended & NP0578A, OE4634F, HQ3141A \\
\hline $\operatorname{rrnAC} 2440$ & 684 & 780 & Shortened & NP0956A, OE4360R, HQ3733A \\
\hline $\operatorname{rrnAC} 2460$ & 1,977 & 2,127 & Shortened & NP1264A, HQ3402A, OE4140R \\
\hline $\operatorname{rrnAC} 2469$ & 1,299 & 1,422 & Shortened & HQ2809A, HQ2192A \\
\hline $\operatorname{rrnAC} 2473$ & 1,524 & 1,569 & Shortened & OE2133R, NP3020A \\
\hline $\operatorname{rrnAC} 2474$ & 288 & 351 & Shortened & NP1258A, OE4136R, HQ3399A \\
\hline $\operatorname{rrnAC} 2476$ & 936 & 582 & Extended & NP1312A, OE4133R \\
\hline $\operatorname{rrnAC} 2518$ & 1,266 & 1,221 & Extended & NP1318A, OE3943R, HQ3056A \\
\hline $\operatorname{rrnAC} 2525$ & 1,026 & 735 & Extended & HQ1021A, OE4201R \\
\hline $\operatorname{rrnAC} 2526$ & 390 & 477 & Shortened & NP1272A, HQ2001A, OE4217R \\
\hline $\operatorname{rrnAC} 2529$ & 741 & 906 & Shortened & NP1268A, OE4218F, HQ1025A \\
\hline $\operatorname{rrnAC} 2532$ & 2,766 & 3,069 & Shortened & NP0538A, OE1272R, HQ1460A \\
\hline $\operatorname{rrnAC} 2550$ & 2,154 & 2,241 & Shortened & OE1267R, NP0536A, HQ1456A \\
\hline $\operatorname{rrnAC} 2558$ & 1,863 & 1,443 & Extended & OE3889R, HQ3102A, NP1576A \\
\hline $\operatorname{rrnAC} 2565$ & 978 & 1,038 & Shortened & HQ3671A, NP0900A, OE4195F \\
\hline
\end{tabular}


Table 2 continued

\begin{tabular}{|c|c|c|c|c|}
\hline ORF & $\begin{array}{l}\text { Corrected } \\
\text { length (aa) }\end{array}$ & $\begin{array}{l}\text { Original } \\
\text { length (aa) }\end{array}$ & $\begin{array}{l}\text { Direction } \\
\text { of change }\end{array}$ & Homologous ORFs \\
\hline $\operatorname{rrnAC} 2582$ & 699 & 870 & Shortened & NP1406A, HQ1040A, OE4235F \\
\hline $\operatorname{rrnAC} 2586$ & 1,200 & 1,065 & Extended & HQ3704A, NP1412A, OE4236F \\
\hline $\operatorname{rrnAC} 2592$ & 975 & 1,281 & Shortened & HQ1035A, NP1818A \\
\hline $\operatorname{rrnAC} 2627$ & 2,061 & 2,106 & Shortened & NP1344A, HQ2213A \\
\hline $\operatorname{rrnAC} 2629$ & 804 & 864 & Shortened & NP5160A \\
\hline $\operatorname{rrnAC} 2630$ & 858 & 993 & Shortened & OE4085R, NP0606A, HQ3650A \\
\hline $\operatorname{rrnAC} 2633$ & 1,356 & 888 & Extended & rrnAC0404, OE3070R \\
\hline $\operatorname{rrnAC} 2636$ & 624 & 720 & Shortened & NP5088A, OE3906F \\
\hline $\operatorname{rrnAC} 2642$ & 738 & 246 & Extended & NP5114A, HQ2624A, OE2740F \\
\hline $\operatorname{rrnAC} 2656$ & 1,125 & 1,182 & Shortened & HQ2450A, OE2317R \\
\hline $\operatorname{rrnAC} 2657$ & 1,194 & 1,062 & Extended & OE5132F, rrnB0290, NP1412A \\
\hline $\operatorname{rrnAC} 2714$ & 1,569 & 1,179 & Extended & NP0482A, HQ1003A, OE4390F \\
\hline $\operatorname{rrnAC} 2722$ & 510 & 558 & Shortened & NP0462A, HQ3640A, OE4429F \\
\hline $\operatorname{rrnAC} 2748$ & 435 & 582 & Shortened & NP5152A, HQ3265A, OE4027F \\
\hline $\operatorname{rrnAC} 2749$ & 453 & 186 & Extended & NP5150A, OE4028R, HQ3266A \\
\hline $\operatorname{rrnAC} 2753$ & 648 & 687 & Shortened & HQ1473A \\
\hline $\operatorname{rrnAC} 2754$ & 366 & 417 & Shortened & NP5146A, OE4039F \\
\hline $\operatorname{rrnAC} 2755$ & 1,206 & 1,257 & Shortened & OE4034R, HQ3066A, NP0030A \\
\hline $\operatorname{rrnAC} 2756$ & 2,046 & 1,770 & Extended & NP5144A, HQ3065A, OE4041F \\
\hline $\operatorname{rrnAC} 2761$ & 1,143 & 960 & Extended & OE2170R, HQ2450A \\
\hline $\operatorname{rrnAC} 2772$ & 1,521 & 1,617 & Shortened & NP1074A, HQ2660A \\
\hline $\operatorname{rrnAC} 2776$ & 1,488 & 1,260 & Extended & pNG7305, OE2076F, HQ2506A \\
\hline $\operatorname{rrnAC} 2780$ & 1,356 & 1,443 & Shortened & NP4376A, HQ3643A, OE3922R \\
\hline $\operatorname{rrnAC} 2781$ & 894 & 711 & Extended & NP0190A, OE3921F, HQ3644A \\
\hline $\operatorname{rrnAC} 2782$ & 1,236 & 1,140 & Extended & NP0192A \\
\hline $\operatorname{rrnAC} 2783$ & 1,437 & 1,296 & Extended & NP5292A, OE2063R \\
\hline $\operatorname{rrnAC} 2798$ & 552 & 393 & Extended & OE3905F, HQ1379A, NP0086A \\
\hline $\operatorname{rrnAC} 2800$ & 612 & 699 & Shortened & NP0092A, OE3902R, HQ1377A \\
\hline $\operatorname{rrnAC} 2804$ & 516 & 447 & Extended & NP1700A, OE3895F \\
\hline $\operatorname{rrnAC} 2806$ & 381 & 228 & Extended & NP1698A, HQ1429A, OE3894R \\
\hline $\operatorname{rrnAC} 2810$ & 909 & 990 & Shortened & OE3892R, NP1688A, HQ3137A \\
\hline $\operatorname{rrnAC} 2811$ & 1,905 & 1,128 & Extended & OE3889R, HQ3102A, NP1576A \\
\hline $\operatorname{rrnAC} 2818$ & 1,344 & 1,479 & Shortened & NP2252A, HQ3104A, OE3882R \\
\hline $\operatorname{rrnAC} 2822$ & 951 & 309 & Extended & NP2248A, OE3879F, HQ3106A \\
\hline $\operatorname{rrnAC} 2831$ & 540 & 411 & Extended & NP5076A, HQ1339A, OE3871R \\
\hline $\operatorname{rrnAC} 2834$ & 1,032 & 1,677 & Shortened & NP1066A, OE4144R, HQ1407A \\
\hline $\operatorname{rrnAC} 2836$ & 1,206 & 1,359 & Shortened & HQ2439A, NP3538A \\
\hline $\operatorname{rrnAC} 2851$ & 744 & 786 & Shortened & NP0554A, OE4165R, HQ3686A \\
\hline $\operatorname{rrnAC} 2857$ & 2,196 & 2,238 & Shortened & NP1350A, OE4181R, HQ3684A \\
\hline $\operatorname{rrnAC} 2859$ & 630 & 678 & Shortened & NP1332A, HQ3517A \\
\hline $\operatorname{rrnAC} 2867$ & 1,467 & 1,353 & Extended & OE4370R, NP5292A \\
\hline $\operatorname{rrnAC} 2870$ & 1,092 & 951 & Extended & HQ3382A, OE4359F \\
\hline $\operatorname{rrnAC} 2891$ & 1,698 & 1,773 & Shortened & NP1008A, OE4122R, HQ3049A \\
\hline rrnAC2893 & 975 & 873 & Extended & NP0698A, HQ3439A, OE2975F \\
\hline $\operatorname{rrnAC} 2901$ & 1,728 & 1,278 & Extended & NP0898A, OE4471R \\
\hline $\operatorname{rrnAC} 2933$ & 720 & 558 & Extended & NP0238A, HQ1390A \\
\hline $\operatorname{rrnAC} 2937$ & 2,844 & 2,895 & Shortened & OE1286R, NP0232A \\
\hline $\operatorname{rrnAC} 3005$ & 927 & 999 & Shortened & NP1116A, HQ3034A, OE3214F \\
\hline rrnAC3008 & 1,158 & 723 & Extended & NP1114A, HQ3033A, OE3216F \\
\hline
\end{tabular}


Table 2 continued

\begin{tabular}{|c|c|c|c|c|}
\hline ORF & $\begin{array}{l}\text { Corrected } \\
\text { length (aa) }\end{array}$ & $\begin{array}{l}\text { Original } \\
\text { length (aa) }\end{array}$ & $\begin{array}{l}\text { Direction } \\
\text { of change }\end{array}$ & Homologous ORFs \\
\hline $\operatorname{rrnAC} 3046$ & 1,104 & 1,146 & Shortened & NP0884A, HQ2919A \\
\hline $\operatorname{rrnAC} 3050$ & 2,199 & 2,514 & Shortened & rrnAC0848, rrnAC2044, HQ2141A, NP6028A \\
\hline $\operatorname{rrnAC} 3062$ & 954 & 1,032 & Shortened & rrnAC1698, OE1358R, HQ2259A \\
\hline $\operatorname{rrnAC} 3071$ & 915 & 1,149 & Shortened & OE3959R, HQ3234A, NP5036A \\
\hline $\operatorname{rrnAC} 3074$ & 699 & 963 & Shortened & NP0072A, HQ3236A, OE3964R \\
\hline rrnAC3079 & 573 & 624 & Shortened & NP3402A \\
\hline $\operatorname{rrnAC} 3083$ & 375 & 423 & Shortened & NP0948A, OE4292F, HQ3465A \\
\hline rrnAC3100 & 2,010 & 2,049 & Shortened & NP2262A, HQ1094A, OE3832F \\
\hline $\operatorname{rrnAC} 3121$ & 651 & 1,194 & Shortened & HQ1495A \\
\hline $\operatorname{rrnAC} 3130$ & 939 & 645 & Extended & HQ1618A, rrnB0227 \\
\hline $\operatorname{rrnAC} 3132$ & 1,392 & 1,419 & Shortened & HQ1619A, rrnAC2624 \\
\hline $\operatorname{rrnAC} 3137$ & 1,047 & 798 & Extended & NP0860A, HQ3045A, OE4446R \\
\hline $\operatorname{rrnAC} 3167$ & 687 & 750 & Shortened & OE1188F, rrnAC1953 \\
\hline $\operatorname{rrnAC} 3182$ & 627 & 666 & Shortened & NP3516A, rrnAC1228 \\
\hline $\operatorname{rrnAC} 3198$ & 1,215 & 1,044 & Extended & NP1072A \\
\hline $\operatorname{rrnAC} 3210$ & 1,311 & 1,356 & Shortened & NP4992A, OE3792F, HQ3101A \\
\hline $\operatorname{rrnAC} 3214$ & 864 & 936 & Shortened & HQ3098A, OE3787R, NP2524A \\
\hline rrnAC3226 & 1,671 & 1,599 & Extended & NP5136A \\
\hline $\operatorname{rrnAC} 3236$ & 1,383 & 420 & Extended & OE1018F, rrnAC1586 \\
\hline rrnAC3256 & 546 & 663 & Shortened & NP3054A, HQ3084A, OE3752R \\
\hline $\operatorname{rrnAC} 3268$ & 678 & 498 & Extended & NP5010A, OE3731R, HQ3131A \\
\hline $\operatorname{rrnAC} 3272$ & 981 & 834 & Extended & NP5006A, HQ3129A, OE3735F \\
\hline rrnAC3279 & 1,107 & 1,032 & Extended & NP2398A, OE3722F, HQ3125A \\
\hline rrnAC3302 & 786 & 735 & Extended & OE3439F, HQ2249A \\
\hline $\operatorname{rrnAC} 3328$ & 531 & 621 & Shortened & NP1380A, OE1858F, HQ1684A \\
\hline $\operatorname{rrnAC} 3342$ & 891 & 945 & Shortened & NP4916A, OE3430F, HQ2764A \\
\hline $\operatorname{rrnAC} 3345$ & 453 & 531 & Shortened & HQ1964A, rrnAC2948, OE2717R \\
\hline $\operatorname{rrnAC} 3348$ & 876 & 963 & Shortened & NP4524A, HQ3322A, OE3531R \\
\hline $\operatorname{rrnAC} 3352$ & 609 & 765 & Shortened & NP4518A, OE3537R, HQ2230A \\
\hline rrnAC3371 & 2,361 & 1,971 & Extended & pNG2034, NP3562A, HQ1851A, OE5286R \\
\hline $\operatorname{rrnAC} 3385$ & 573 & 396 & Extended & OE3854R, NP2906A \\
\hline rrnAC3394 & 444 & 525 & Shortened & NP1284A, rrnB0323 \\
\hline $\operatorname{rrnAC} 3420$ & 843 & 885 & Shortened & OE3633R, NP2432A, HQ3036A \\
\hline $\operatorname{rrnAC} 3450$ & 366 & 399 & Shortened & OE3588C1R, NP2486A, HQ3026A \\
\hline $\operatorname{rrnAC} 3452$ & 894 & 951 & Shortened & NP2484A, OE3586R, HQ3027A \\
\hline $\operatorname{rrnAC} 3462$ & 1,929 & 2,019 & Shortened & NP2410A, OE3580R, HQ2579A \\
\hline $\operatorname{rrnAC} 3475$ & 1,113 & 594 & Extended & NP6258A \\
\hline $\operatorname{rrnAC} 3486$ & 636 & 687 & Shortened & NP4286A \\
\hline rrnAC3509 & 624 & 471 & Extended & OE1814R, HQ1569A \\
\hline $\operatorname{rrnAC} 3528$ & 459 & 477 & Shortened & rrnAC3526, pNG2015 \\
\hline rrnAC3536 & 276 & 153 & Extended & NP0840A, OE1853R \\
\hline rrnAC3537 & 1,089 & 681 & Extended & HQ1674A, OE1854R, NP0842A \\
\hline $\operatorname{rrnAC} 3551$ & 1,026 & 750 & Extended & NP2032A, HQ3010A, rrnAC1926, OE5141R \\
\hline rrnB0092 & 1,533 & 1,599 & Shortened & HQ1972A \\
\hline $\operatorname{rrnB} 0172$ & 591 & 507 & Extended & NP1842A \\
\hline rrnB0198 & 1,530 & 1,623 & Shortened & NP0556A, OE4115F \\
\hline $\operatorname{rrnB} 0242$ & 1,173 & 1,287 & Shortened & NP2606A, HQ2307A \\
\hline $\operatorname{rrnB} 0257$ & 834 & 888 & Shortened & NP4244A, OE1942F \\
\hline $\operatorname{rrnB0265}$ & 1,683 & 1,851 & Shortened & NP4242A \\
\hline
\end{tabular}


Table 2 continued

\begin{tabular}{|c|c|c|c|c|}
\hline ORF & $\begin{array}{l}\text { Corrected } \\
\text { length (aa) }\end{array}$ & $\begin{array}{l}\text { Original } \\
\text { length (aa) }\end{array}$ & $\begin{array}{l}\text { Direction } \\
\text { of change }\end{array}$ & Homologous ORFs \\
\hline rrnB0266 & 1,404 & 1,011 & Extended & NP3416A \\
\hline $\operatorname{rrnB} 0275$ & 1,101 & 1,065 & Extended & rrnAC0899, OE4576F \\
\hline $\operatorname{rrnB0325}$ & 1,107 & 1,278 & Shortened & OE5142F, NP2128A, rrnAC3284, HQ3147A \\
\hline pNG2007 & 939 & 1,119 & Shortened & OE4023F, NP1282A, rrnAC2744, HQ3263A \\
\hline pNG2015 & 516 & 615 & Shortened & rrnAC3526, NP1956A \\
\hline pNG4017 & 1,254 & 1,125 & Extended & NP2168A, rrnAC2207, OE2401F \\
\hline pNG4035 & 561 & 516 & Extended & OE3768F, NP5358A \\
\hline pNG5001 & 1,134 & 1,104 & Extended & rrnAC0252, NP0102A, HQ1815A, OE1005F \\
\hline pNG5004 & 1,488 & 1,068 & Extended & $\operatorname{rrnAC} 0250$ \\
\hline pNG5010 & 1,632 & 879 & Extended & OE5248F, HQ1543A, NP2464A \\
\hline pNG5131 & 633 & 579 & Extended & rrnAC3384, OE4753R \\
\hline pNG5139 & 1,251 & 312 & Extended & HQ2051A, NP6268A, OE1070R \\
\hline pNG6047 & 591 & 618 & Shortened & HQ1118A, OE2691R, rrnAC0503, NP2664A \\
\hline pNG6054 & 423 & 1,047 & Shortened & NP5022A \\
\hline pNG6069 & 417 & 444 & Shortened & NP4526A, rrnAC1905 \\
\hline pNG6075 & 378 & 477 & Shortened & OE7144R, NP3058A \\
\hline pNG6092 & 294 & 324 & Shortened & pNG6058, OE7057F, NP3002A, HQ2407A \\
\hline pNG6120 & 861 & 921 & Shortened & OE5424R \\
\hline pNG6141 & 615 & 585 & Extended & OE5415R \\
\hline pNG7012 & 1,281 & 1,026 & Extended & OE1077R, rrnAC3239, HQ2680A, NP2322A \\
\hline pNG7037 & 1,488 & 1,725 & Shortened & NP5056A \\
\hline pNG7040 & 1,182 & 999 & Extended & pNG7041, OE4576F \\
\hline pNG7050 & 750 & 705 & Extended & HQ3696A, rrnAC0479, NP1198A, OE3661F \\
\hline pNG7058 & 528 & 501 & Extended & OE1252R, HQ2374A, NP1606A \\
\hline pNG7060 & 1,272 & 1,302 & Shortened & NP6204A \\
\hline pNG7066 & 1,017 & 1,107 & Shortened & OE2128F, HQ2746A, NP1386A \\
\hline pNG7078 & 1,071 & 1,242 & Shortened & NP1388A, HQ1592A \\
\hline pNG7081 & 399 & 363 & Extended & HQ4010A \\
\hline pNG7101 & 1,971 & 2,004 & Shortened & HQ1729A \\
\hline pNG7106 & 897 & 819 & Extended & OE2497F, HQ2422A, NP1346A \\
\hline pNG7178 & 984 & 834 & Extended & HQ2189A \\
\hline pNG7227 & 747 & 612 & Extended & NP0054A, HQ1091A, OE3843F \\
\hline pNG7244 & 2,481 & 2,166 & Extended & pNG7246, HQ1944A \\
\hline pNG7252 & 1,659 & 1,788 & Shortened & OE2316R, rrnAC2655, HQ2451A \\
\hline pNG7278 & 1,041 & 1,155 & Shortened & OE4674F, HQ1124A \\
\hline pNG7280 & 540 & 489 & Extended & pNG6134, NP5298A \\
\hline pNG7297 & 603 & 630 & Shortened & NP0672A \\
\hline pNG7321 & 381 & 408 & Shortened & HQ1769A, pNG7235, OE3930R, NP0566A \\
\hline pNG7327 & 1,512 & 1,572 & Shortened & HQ1784A, NP0802A, OE1568F \\
\hline pNG7342 & 1,098 & 1,128 & Shortened & pNG7026, OE5170F \\
\hline pNG7351 & 1,065 & 1,089 & Shortened & rrnAC0191, NP1260A, OE4674F, HQ3648A \\
\hline pNG7377 & 432 & 324 & Extended & HQ3372A \\
\hline pNG7380 & 1,746 & 1,932 & Shortened & HQ1768A \\
\hline
\end{tabular}

Using our semiautomatic checking procedure, candidate genes with probable errors in start codon assignment were identified and subjected to manual curation. When sufficiently strong evidences were found, the start codon was reassigned using the manual curation options of HaloLex. For each gene in the list (first column), we provide the corrected (second column) and original length (third column) of the aminoacid sequence, and the set of homologous genes that support our decision for the new start codon assignment (fifth column). The redundant fourth column facilitates a quick overview of whether sequences were extended or shortened with respect to their original length 
curation, as it does not only support detailed analysis but, once a decision is taken, allows it to be conveniently made persistent with a few clicks.

\section{Conclusions and outlook}

We have described HaloLex, a software system for the central management, integration, and web-based visualization of genomic and other -omics data. A number of HaloLex functionalities are specifically tailored to halophilic archaea, but the system can handle any given microorganism.

HaloLex has proven an indispensable tool for the data management, curation, and in-depth bioinformatic analysis of three halophilic archaea sequenced in-house, namely Halobacterium salinarum (strain R1), Natronomonas pharaonis, and Haloquadratum walsbyi. HaloLex summarizes all available data for a given organism including experimental data, like, e.g., proteomics, in an easy-to-use web interface. This proved to be of enormous importance for both, the daily user of genome information as well as for the manual curator of the gene annotation in these organisms.

In this article, we further reviewed a number of selected, biologically relevant results we obtained for these species, thus highlighting the capabilities of HaloLex for prediction and curation of gene assignment, in particular by the integrated analysis of genomic with proteomic data.

Lately, we have applied HaloLex functionalities to the published genome of another halophilic archaeon, Haloarcula marismortui, which resulted in a significantly improved version of the original gene prediction.

Other halophiles (also from the bacterial kingdom) like Halobacillus halophilus are currently being annotated by different collaborations, which shows that HaloLex could be a useful tool also for a broader user-community. Based on our promising experiences, we thus encourage potential collaborators to consider employing our HaloLex server as a data repository and a tool for curation and analysis of their genomes (and proteomes, etc.) of interest. At the same time, HaloLex would allow such groups to make their data available to the public (or restricted user groups) without having to take up the burden of developing and hosting their own software and hardware infrastructure.

Our ongoing and future activities are focussed on making those data and methods fully available, which so far can be used only internally (e.g., data from transcriptomics experiments). Moreover, HaloLex functionalities are continuously being improved and extended. Currently, we are about to couple software modules for text-mining and metabolic modeling, which we are developing in our group to the HaloLex web application. We also plan to release our web-service interface to support mining of HaloLex data over the Internet.

Acknowledgments We thank Volker Hickmann and Jan Wolfertz who were involved in early phases of the HaloLex project, and Karin Gross who has recently joined the team. Development of the Halolex system benefited greatly from the MIGenAS infrastructure for bioinformatics software and data, which was developed with funds from the Max-Planck-Society. Part of this work was funded by the Deutsche Forschungsgemeinschaft within the priority program SP1112.

Open Access This article is distributed under the terms of the Creative Commons Attribution Noncommercial License which permits any noncommercial use, distribution, and reproduction in any medium, provided the original author(s) and source are credited.

\section{References}

Aivaliotis M, Gevaert K, Falb M, Tebbe A, Konstantinidis K, Bisle B, Klein C, Martens L, Staes A, Timmerman E, Van Damme J, Siedler F, Pfeiffer F, Vandekerckhove J, Oesterhelt D (2007) Large-scale identification of $\mathrm{N}$-terminal peptides in the halophilic archaea Halobacterium salinarum and Natronomonas pharaonis. J Proteome Res 6:2195-2204

Altschul SF, Madden TL, Schaffer AA, Zhang J, Zhang Z, Miller W, Lipman DJ (1997) Gapped BLAST and PSI-BLAST: a new generation of protein database search programs. Nucleic Acids Res 25:3389-3402

Baliga NS, Bonneau R, Facciotti MT, Pan M, Glusman G, Deutsch EW, Shannon P, Chiu Y, Weng RS, Gan RR, Hung P, Date SV, Marcotte E, Hood L, Ng WV (2004) Genome sequence of Haloarcula marismortui: a halophilic archaeon from the Dead Sea. Genome Res 14:2221-2234

Bisle B, Schmidt A, Scheibe B, Klein C, Tebbe A, Kellermann J, Siedler F, Pfeiffer F, Lottspeich F, Oesterhelt D (2006) Quantitative profiling of the membrane proteome in a halophilic archaeon. Mol Cell Proteomics 5:1543-1558

Bolhuis H, Palm P, Wende A, Falb M, Rampp M, Rodriguez-Valera F, Pfeiffer F, Oesterhelt D (2006) The genome of the square archaeon Haloquadratum walsbyi: life at the limits of water activity. BMC Genomics 7:169

Bryson K, Loux V, Bossy R, Nicolas P, Chaillou S, van de Guchte M, Penaud S, Maguin E, Hoebeke M, Bessieres P, Gibrat JF (2006) AGMIAL: implementing an annotation strategy for prokaryote genomes as a distributed system. Nucleic Acids Res 34:35333545

Dambeck M, Soppa J (2008) Characterization of a Haloferax volcanii member of the enolase superfamily: deletion mutant construction, expression analysis, and transcriptome comparison. Arch Microbiol. doi:10.1007/s00203-008-0379-1

Falb M, Pfeiffer F, Palm P, Rodewald K, Hickmann V, Tittor J, Oesterhelt D (2005) Living with two extremes: conclusions from the genome sequence of Natronomonas pharaonis. Genome Res 15:1336-1343

Falb M, Aivaliotis M, Garcia-Rizo C, Bisle B, Tebbe A, Klein C, Konstantinidis K, Siedler F, Pfeiffer F, Oesterhelt D (2006) Archaeal N-terminal protein maturation commonly involves $\mathrm{N}$ terminal acetylation: a large-scale proteomics survey. J Mol Biol 362:915-924

Falb M, Müller K, Königsmaier L, Oberwinkler T, Horn P, von Gronau S, Gonzalez O, Pfeiffer F, Bornberg-Bauer E, Oesterhelt 
D (2008) Metabolism of halophilic archaea. Extremophiles 12:177-196

Gonzalez O, Gronau S, Falb M, Pfeiffer F, Mendoza E, Zimmer R, Oesterhelt D (2008) Reconstruction, modeling \& analysis of Halobacterium salinarum R-1 metabolism. Mol Biosyst 4:148159

Kall L, Krogh A, Sonnhammer EL (2004) A combined transmembrane topology and signal peptide prediction method. J Mol Biol 338:1027-1036

Karlin S, Brocchieri L, Trent J, Blaisdell BE, Mrazek J (2002) Heterogeneity of genome and proteome content in bacteria, archaea, and eukaryotes. Theor Popul Biol 61:367-390

Keese PK, Gibbs A (1992) Origins of genes: "big bang" or continuous creation? Proc Natl Acad Sci USA 89:9489-9493

Kitano H (2002) Systems biology: a brief overview. Science 295:1662-1664

Klein C, Garcia-Rizo C, Bisle B, Scheffer B, Zischka H, Pfeiffer F, Siedler F, Oesterhelt D (2005) The membrane proteome of Halobacterium salinarum. Proteomics 5:180-197

Klein C, Aivaliotis M, Olsen JV, Falb M, Besir H, Scheffer B, Bisle B, Tebbe A, Konstantinidis K, Siedler F, Pfeiffer F, Mann M, Oesterhelt D (2007) The low molecular weight proteome of Halobacterium salinarum. J Proteome Res 6(4):1510-1518

Konstantinidis K, Tebbe A, Klein C, Scheffer B, Aivaliotis M, Bisle B, Falb M, Pfeiffer F, Siedler F, Oesterhelt D (2007) Genomewide proteomics of Natronomonas pharaonis. J Proteome Res 6:185-193

Markowitz VM, Korzeniewski F, Palaniappan K, Szeto E, Werner G, Padki A, Zhao X, Dubchak I, Hugenholtz P, Anderson I, Lykidis A, Mavromatis K, Ivanova N, Kyrpides NC (2006) The integrated microbial genomes (IMG) system. Nucleic Acids Res 34:D344-348

McHardy AC, Goesmann A, Pühler A, Meyer F (2004) Development of joint application strategies for two microbial gene finders. Bioinformatics 20:1622-1631

Meyer F, Goesmann A, McHardy AC, Bartels D, Bekel T, Clausen J, Kalinowski J, Linke B, Rupp O, Giegerich R, Pühler A (2003) GenDB - an open source genome annotation system for prokaryote genomes. Nucleic Acids Res 31:2187-2195

Ng WV, Kennedy SP, Mahairas GG, Berquist B, Pan M, Shukla HD, Lasky SR, Baliga NS, Thorsson V, Sbrogna J, Swartzell S, Weir D, Hall J, Dahl TA, Welti R, Goo YA, Leithauser B, Keller K, Cruz R, Danson MJ, Hough DW, Maddocks DG, Jablonski PE, Krebs MP, Angevine CM, Dale H, Isenbarger TA, Peck RF, Pohlschroder M, Spudich JL, Jung KW, Alam M, Freitas T, Hou S, Daniels CJ, Dennis PP, Omer AD, Ebhardt H, Lowe TM, Liang P, Riley M, Hood L, DasSarma S (2000) Genome sequence of Halobacterium species NRC-1. Proc Natl Acad Sci USA 97:12176-12181

Nielsen P, Krogh A (2005) Large-scale prokaryotic gene prediction and comparison to genome annotation. Bioinformatics 21:43224329

Pfeiffer F, Schuster SC, Broicher A, Falb M, Palm P, Rodewald K, Ruepp A, Soppa J, Tittor J, Oesterhelt D (2008) Evolution in the laboratory: the genome of Halobacterium salinarum strain R1 compared to that of strain NRC-1. Genomics 91:335-346
Pleissner KP, Eifert T, Buettner S, Schmidt F, Boehme M, Meyer TF, Kaufmann SH, Jungblut PR (2004) Web-accessible proteome databases for microbial research. Proteomics 4:1305-1313

Rampp M, Soddemann T, Lederer H (2006) The MIGenAS integrated bioinformatics toolkit for web-based sequence analysis. Nucleic Acids Res 34:W15-W19

Rattei T, Arnold R, Tischler P, Lindner D, Stumpflen V, Mewes HW (2006) SIMAP: the similarity matrix of proteins. Nucleic Acids Res 34:D252-D256

Riley ML, Schmidt T, Artamonova II, Wagner C, Volz A, Heumann K, Mewes HW, Frishman D (2007) PEDANT genome database: 10 years online. Nucleic Acids Res 35:D354-D357

Rose RW, Bruser T, Kissinger JC, Pohlschröder M (2002) Adaptation of protein secretion to extremely high-salt conditions by extensive use of the twin-arginine translocation pathway. Mol Microbiol 45:943-950

Scheuch S, Marschaus L, Sartorius-Neef S, Pfeifer F (2008) Regulation of gvp genes encoding gas vesicle proteins in halophilic Archaea. Arch Microbiol. doi:10.1007/s00203-008-0362-x

Schneider KL, Pollard KS, Baertsch R, Pohl A, Lowe TM (2006) The UCSC Archaeal Genome Browser. Nucleic Acids Res 34:D407D410

Sharp PM, Li WH (1987) The codon adaptation index-a measure of directional synonymous codon usage bias, and its potential applications. Nucleic Acids Res 15:1281-1295

Soppa J, Baumann A, Brenneis M, Dambeck M, Hering O, Lange C (2008) Genomics and functional genomics with haloarchaea. Arch Microbiol. doi:10.1007/s00203-008-0376-4

Stearns J, Chinnici R, Sahoo (2006) An Introduction to the Java EE 5 Platform. http://java.sun.com/developer/technicalArticles/J2EE/ intro_ee5/

Stein L (2002) Creating a bioinformatics nation. Nature 417:119-120

Tarasov VY, Besir H, Schwaiger R, Klee K, Furtwängler K, Pfeiffer F, Oesterhelt D (2008) A small protein from the bop-brp intergenic region of Halobacterium salinarum contains a zinc finger motif and regulates bop and crtB1 transcription. Mol Microbiol 67:772-780

Tebbe A, Klein C, Bisle B, Siedler F, Scheffer B, Garcia-Rizo C, Wolfertz J, Hickmann V, Pfeiffer F, Oesterhelt D (2005) Analysis of the cytosolic proteome of Halobacterium salinarum and its implication for genome annotation. Proteomics 5:168179

Teufel K, Bleiholder A, Griesbach T, Pfeifer F (2008) Variations in the multiple tbp genes in different Halobacterium salinarum strains and their expression during growth. Arch Microbiol. doi: 10.1007/s00203-008-0383-5

Twellmeyer J, Wende A, Wolfertz J, Pfeiffer F, Panhuysen M, Zaigler A, Soppa J, Welzl G, Oesterhelt D (2007) Microarray analysis in the Archaeon Halobacterium salinarum strain R1. PLoS ONE 2:e1064

Veloso F, Riadi G, Aliaga D, Lieph R, Holmes DS (2005) Largescale, multi-genome analysis of alternate open reading frames in bacteria and archaea. Omics 9:91-105

Wu CH, Huang H, Nikolskaya A, Hu Z, Barker WC (2004) The iProClass integrated database for protein functional analysis. Comput Biol Chem 28:87-96 